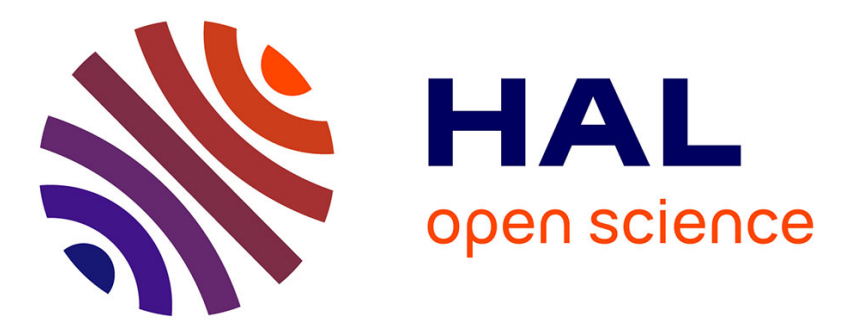

\title{
A discontinuous Galerkin Trefftz type method for solving the two dimensional Maxwell equations
}

Håkon Sem Fure, Sébastien Pernet, Margot Sirdey, Sébastien Tordeux

\section{To cite this version:}

Håkon Sem Fure, Sébastien Pernet, Margot Sirdey, Sébastien Tordeux. A discontinuous Galerkin Trefftz type method for solving the two dimensional Maxwell equations. SN Partial Differential Equations and Applications, 2020, 1 (23), pp.19. 10.1007/s42985-020-00024-0 . hal-02899350

\section{HAL Id: hal-02899350 https://hal.inria.fr/hal-02899350}

Submitted on 15 Jul 2020

HAL is a multi-disciplinary open access archive for the deposit and dissemination of scientific research documents, whether they are published or not. The documents may come from teaching and research institutions in France or abroad, or from public or private research centers.
L'archive ouverte pluridisciplinaire HAL, est destinée au dépôt et à la diffusion de documents scientifiques de niveau recherche, publiés ou non, émanant des établissements d'enseignement et de recherche français ou étrangers, des laboratoires publics ou privés. 


\title{
A discontinuous Galerkin Trefftz type method for solving the two dimensional Maxwell equations
}

\author{
Håkon Sem Fure - Sébastien Pernet • Margot Sirdey • \\ Sébastien Tordeux
}

Received: date / Accepted: date

\begin{abstract}
Trefftz methods are known to be very efficient to reduce the numerical pollution when associated to plane wave basis. However, these local basis functions are not adapted to the computation of evanescent modes or corner singularities. In this article, we consider a two dimensional time-harmonic Maxwell system and we propose a formulation which allows to design an electromagnetic Trefftz formulation associated to local Galerkin basis computed thanks to an auxiliary Nédélec finite element method. The results are illustrated with numerous numerical examples. The considered test cases reveal that the short range and long range propagation phenomena are both well taken into account.
\end{abstract}

Keywords Trefftz method · Electromagnetic Wave · Nédélec Finite Element · Numercial Methods · Transverse Electric Polarization · Maxwell equation

\section{Introduction}

Numerical methods like the Finite Element Method (FEM), see [3, 26, 32, and the Finite Difference Method (FDM), see 41] for example, are widely used to solve time-harmonic electromagnetic wave equations. One limitation they all face is called the pollution effect. When considering the numerical solution of a propagation phenomenon with wavenumbers $k$ posed on domains with length $L$, the numerical accuracy deteriorates when $k L$ becomes large. This has been highlighted in the following articles [24, 25. This phenomenon is related to a numerical dispersion and is called numerical pollution. A detailed analysis with error estimates has been proposed in [30]. This issue is of particular importance at high frequency or on large domains where the number of degrees of freedom per wavelength should be chosen large to achieve a given accuracy.

Classically, numericians resort to one or more of the following remedies, which can be combined. The first one consists in considering high-order FEM, see for example 2]. A second answer to the numerical pollution issue is Discontinuous Galerkin (DG) finite elements that are less dispersive [1. This latter approach can be of particular interest in the context of inverse problems.

The German mathematician Erich Trefftz proposed a paradigm in which basis functions are taken to be local solutions to the partial differential equations system of interest. The intuition behind this idea is that such basis functions can better approximate physical phenomena, and be especially less dispersive than classical polynomial basis functions. Trefftz methods gather simultaneously the possibility of being flexible like highorder method and of being less dispersive at low-order as pointed out in 40 .

Another important feature of Trefftz methods relies on the difficulty to solve wave problems on huge domains due to memory limitation. This fact is less relevant in homogeneous media where accelerated Boundary Element Method (BEM) [12, 38, 45] or integral equation collocation method [6] can be used. A natural idea is also to resort to a domain decomposition method to successively deal with different subdomains. Trefftz formalism, especially in the context of the Ultra-Weak Variational Formulation (UWVF) [7, 9, 10, 13, 43, seems to be one of the most relevant solutions.

Moreover, Trefftz methods have also a variety of formulations which offer a high flexibility. In addition to the UWVF method and to the approach based on the reciprocity principle considered in this paper, the least square method [16] is another Trefftz method which is of great interest. On the other hand, the partition of

Håkon Sem Fure · Sébastien Pernet · Margot Sirdey

ONERA, DTIS, 2 avenue Edouard Belin 31000 Toulouse

E-mail: sebastien.pernet@onera.fr, margot.sirdey@onera.fr

Margot Sirdey · Sébastien Tordeux

Universite de Pau et des Pays de l'Adour, E2S-UPPA, CNRS, INRIA, équipe Magique 3D, LMAP, Pau, France

E-mail: sebastien.tordeux@univ-pau.fr, margot.sirdey@onera.fr 
unity method 4] shares also some similarities with Trefftz methods even if local basis functions are not exact solutions of Maxwell equations. This is particularly true for problems involving corner singularities but this subject is out of scope for our paper.

In the context of long range propagation, most Trefftz methods are associated to plane wave basis [15, 17. 18, 20, 31, or other analytical solution like Bessel functions. Hiptmair et al. have contributed significantly in this paradigm on the theoretical level. In particular, a good starting point would be [19] which is a survey of Trefftz type methods. The main drawback of these methods is that they are associated to ill-conditioned linear system which leads in double precision to a lack of accuracy. So much so that in [11, 27, methods improving linear system properties have been presented. Moreover, these types of basis approximate accurately only a few kind of wave functions. For example, plane wave basis functions have difficulties to approximate evanescent modes, corner singularities or transitions between light and shadows which are located behind obstacles. In some sense these methods have the same limitation than geometrical optic methods and have difficulties to compute near field accurately.

An important advance was proposed in [21, 22] where the local solutions were not analytical but constructed thanks to a local solver, which was in this case a BEM. This has led to a method where all kinds of waves are correctly computed. A similar idea was later developed in 5 where a symmetric Trefftz method was associated to a local BEM to compute solutions of the Helmholtz equation in two dimensions. In the latter paper, it was remarked that an accurate resolution of the local solver impressively increases the global accuracy. This is rather surprising since this improvement does not cause any extra cost. Our approach is comparable to static condensation methods [44] and to hybridizable discontinuous Galerkin methods [36] where interior degrees of freedom are eliminated from the final system. However, a local BEM solver is not always appropriate. It cannot easily handle heterogeneous or anisotropic domains. BEM is also less popular in the numerical simulation community. In this paper, the local basis will be computed thanks to a local FEM solver. A wide class of wave equations will then be studied. To highlight this last aspect, we have considered electromagnetic wave propagation in heterogeneous media.

In the present paper, we propose to parameterize the local Trefftz basis functions by an impedance condition (also called Fourier-Robin condition). This is a second improvement of 5$]$ where the elements should be included in a strip of width of half a wavelength to avoid numerical resonance frequencies. This enables to consider a wide class of elements with no size restriction.

The objective of this paper is to develop a Trefftz electromagnetic method and to investigate its performance in particular with respect to the pollution effect. It is structured as follows. Section 1 reviews Maxwell equations and introduce the second-order Maxwell problem and its hypotheses. Next, our Trefftz scheme is elaborated in Section 2. The associated continuous formulation is provided on a general mesh. Section 3 is devoted to the discretization of this latter formulation, which leads to approximations of both Trefftz spaces and local Trefftz basis functions. An example of FEM local solver is presented and uses Nédélec FEM. The final section relates results from our Trefftz method implementation. Numerical error analysis will illustrate improvements on the pollution effect. Examples of wave propagation on different domains will show the method's robustness and accuracy.

\section{Model problem: the simplified Maxwell equations}

The Maxwell system models the propagation of an electromagnetic wave. It reads, in absence of charges and currents and for an isotropic linear medium ${ }^{1}$.

$$
\left\{\begin{array}{lll}
\nabla \cdot \mathbf{d}=0, & \nabla \times \mathbf{e}=-\frac{\partial \mathbf{b}}{\partial t}, & \mathbf{d}=\varepsilon_{0} \varepsilon_{r} \mathbf{e} \\
\nabla \cdot \mathbf{b}=0, & \nabla \times \mathbf{h}=\frac{\partial \mathbf{d}}{\partial t}, & \mathbf{b}=\mu_{0} \mu_{r} \mathbf{h}
\end{array}\right.
$$

where $\varepsilon_{0}\left(\right.$ resp. $\left.\varepsilon_{r}\right)$ and $\mu_{0}\left(\right.$ resp. $\left.\mu_{r}\right)$ are the permittivity (resp. relative permittivity) and the permeability (resp. relative permeability) of the vacuum (resp. of the medium).

This system involves the electric and magnetic field intensities $\mathbf{e}$ and $\mathbf{h}$ and the electric displacement and the magnetic induction $\mathbf{d}$ and $\mathbf{b}$. In this article we suppose that all these fields are time-harmonic. They can therefore be represented by four complex valued functions:

$$
\begin{cases}\mathbf{e}(\mathbf{x}, t)=\mathcal{R}(\exp (-i \omega t) \mathbf{E}(\mathbf{x})), & \mathbf{h}(\mathbf{x}, t)=\mathcal{R}(\exp (-i \omega t) \mathbf{H}(\mathbf{x})) \\ \mathbf{d}(\mathbf{x}, t)=\mathcal{R}(\exp (-i \omega t) \mathbf{D}(\mathbf{x})), & \mathbf{b}(\mathbf{x}, t)=\mathcal{R}(\exp (-i \omega t) \mathbf{B}(\mathbf{x}))\end{cases}
$$

\footnotetext{
1 All along this paper, bold terms refer to either vectors or vectorial functions.
} 
where $\omega$ is the angular frequency that accounts for time-harmonic dependency. It leads to the following timeharmonic Maxwell system:

$$
\left\{\begin{array}{lll}
\nabla \cdot \mathbf{D}=0, & \nabla \times \mathbf{E}=-i \omega \mathbf{B}, & \mathbf{D}=\varepsilon_{0} \varepsilon_{r} \mathbf{E} \\
\nabla \cdot \mathbf{B}=0, & \nabla \times \mathbf{H}=i \omega \mathbf{D}, & \mathbf{B}=\mu_{0} \mu_{r} \mathbf{H}
\end{array}\right.
$$

We suppose, moreover, that the propagation domain $\Omega \times \mathbb{R}$, with $\Omega \subset \mathbb{R}^{2}$, is invariant in the $z$-direction. We consider only the transverse electric polarization:

$$
\begin{cases}\mathbf{E}(\mathbf{x})=E_{x}(x, y) \mathrm{e}_{x}+E_{y}(x, y) \mathrm{e}_{y}, & \mathbf{D}(\mathbf{x})=D_{x}(x, y) \mathrm{e}_{x}+D_{y}(x, y) \mathrm{e}_{y} \\ \mathbf{B}(\mathbf{x})=B_{z}(x, y) \mathrm{e}_{z}, & \mathbf{H}(\mathbf{x})=H_{z}(x, y) \mathrm{e}_{z} .\end{cases}
$$

In this paper, we assume that the computational domain $\Omega \subset \mathbb{R}^{2}$ is a connected domain with polygonal boundary, see Fig. 1. This is representative of most applications, see Fig 11 for an example. Moreover, we assume that $\Omega$ can be decomposed into $P$ subdomains denoted $\Omega_{p}$ i.e $\bar{\Omega}=\bigcup_{p=1, \ldots, P} \bar{\Omega}_{p}$ and $\Omega_{p} \cap \Omega_{q}=\emptyset$, if $p \neq q$, such that $\varepsilon_{r}$ and $\mu_{r}$ are constant on each subdomain.

Finally, we consider the following second-order Maxwell equation subjected to an impedance boundary condition:

$$
\begin{array}{cc}
\nabla \times\left(\frac{1}{\mu_{r}} \nabla \times \mathbf{E}\right)-k_{0}^{2} \varepsilon_{r} \mathbf{E}=0 & \text { in } \Omega, \\
i k_{0} Y \mathbf{n} \times(\mathbf{E} \times \mathbf{n})-\mathbf{n} \times\left(\frac{1}{\mu_{r}} \nabla \times \mathbf{E}\right)=\mathbf{h} & \text { on } \partial \Omega,
\end{array}
$$

where

$-c_{0}=\frac{1}{\sqrt{\varepsilon_{0} \mu_{0}}}$ and $k_{0}=\frac{\omega}{c_{0}}$ are the wave speed of light and the wavenumber in vacuum respectively,

- the function $Y \in L^{\infty}(\partial \Omega)$ is an admittance coefficient with strictly non-positive real part,

- the normal $\mathbf{n} \in \mathbb{R}^{2}$ is the outward unit normal to $\partial \Omega$,

- the boundary term $\mathbf{h}: \partial \Omega \longrightarrow \mathbb{C}^{2}$ is taken to be a purely tangential function in the functional space

$$
L_{t}^{2}(\partial \Omega):=\left\{\mathbf{u} \in\left(L^{2}(\partial \Omega)\right)^{2}, \quad \mathbf{u} \cdot \mathbf{n}=0\right\},
$$

- the solution of this problem is in the following classical Sobolev space $H(\nabla \times, \Omega)$

$$
H(\nabla \times, \Omega)=\left\{\boldsymbol{\omega}: \Omega \rightarrow \mathbb{C}^{2}, \int_{\Omega}|\boldsymbol{\omega}|^{2} d x<\infty, \int_{\Omega}|\nabla \times \boldsymbol{\omega}|^{2} d x<\infty\right\} .
$$

Remark 1 In this paper, we consider a two dimensional problem. However, this problem can be seen as a three dimensional problem on $\Omega \times \mathbb{R}$ whose solution is independent of the $z$-component. The two dimensional vector field $\mathbf{E}$ is composed of the two first components of a three dimensional electromagnetic field problem which is independent of $z$ and with no $z$-component. Equivalently, $\mathbb{C}^{2}$-vectors $\left(E_{x}, E_{y}\right)$ are identified to $\mathbb{C}^{3}$-vectors $\left(E_{x}, E_{y}, 0\right)$ and $H_{z} \in \mathbb{C}$ to $\mathbb{C}^{3}$-vectors $\left(0,0, H_{z}\right)$. In this context, the $\nabla \times$ operators acting on vector fields or on scalar fields are defined as

$$
\nabla \times \mathbf{E}=\partial_{x} E_{y}-\partial_{y} E_{x} \quad \text { and } \quad \nabla \times H_{z}=\left(\partial_{y} H_{z},-\partial_{x} H_{z}\right) .
$$

In the same way, $\mathbf{n} \times$ operators are given by

$$
\mathbf{n} \times \mathbf{E}=n_{x} E_{y}-n_{y} E_{x} \quad \text { and } \quad \mathbf{n} \times H_{z}=\left(n_{y} H_{z},-n_{x} H_{z}\right) .
$$

These hypotheses being considered, (5) is well-posed in the Hadamard sense. For proof in the context of homogeneous media, we refer to [8, 32]. Physical interpretation of the impedance boundary condition can also be found in [39].

This model is simple enough for numerical implementation, and sufficiently rich to perform a comparison with different numerical methods. 


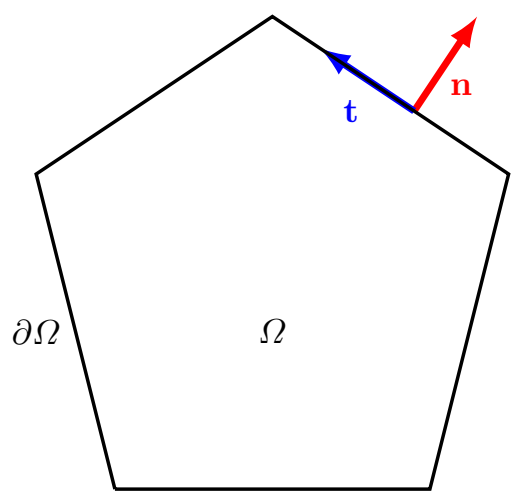

Fig. 1 Schematic view of the computational domain $\Omega$. The domain boundary is denoted by $\partial \Omega$. The unit normal and tangent vectors are represented in red and blue respectively at one point of the boundary.

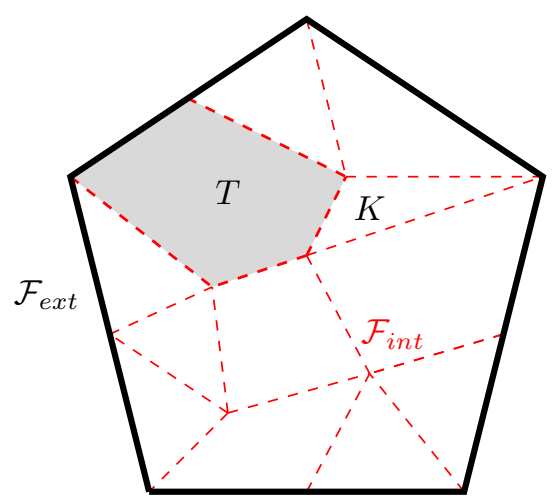

Fig. 2 An example of Trefftz mesh. Interior (resp. exterior) faces are denoted by $\mathcal{F}_{\text {int }}\left(\right.$ resp. $\left.\mathcal{F}_{\text {ext }}\right)$, see dashed red segments (resp. bold segments). Two neighboring macroelements are for example $K$ and $T$.

\section{Construction of a Trefftz scheme}

\subsection{Notations and definitions}

The domain $\Omega$ is meshed by non-overlapping polygonal elements $T$. These open sets are called macroelements, see Fig. 2. The mesh does not need to include only triangles or quadrangles (see the colored pentagon in Fig. 2 and allows to consider a large variety of geometrical configurations. The set of all macro-elements $T$ is denoted by $\mathcal{T}$ and respects the partition of $\Omega$. In other words, one element $T \in \mathcal{T}$ cannot intersect two different subdomains $\Omega_{p}, p=1, \ldots, P$, at the same time. In particular, since there exists a unique $p_{0}=1, \ldots, P$ such that $T \subset \Omega_{p_{0}}, \varepsilon_{r}$ and $\mu_{r}$ are constant on each macro-element $T$.

All along this paper, we call edges of an element $T$ as faces and we define the following sets:

- the set $\mathcal{F}_{\text {int }}$ of interior macro-faces:

$$
\mathcal{F}_{\text {int }}:=\{\partial T \cap \partial K: T, K \in \mathcal{T} \text { with } T \neq K \text { and length }(\partial T \cap \partial K) \neq 0\},
$$

where length $(I)$ refers to the length of the segment $I$ (that is zero for isolated points),

- the set $\mathcal{F}_{\text {ext }}$ of exterior macro-faces:

$$
\mathcal{F}_{\text {ext }}:=\{\partial T \cap \partial \Omega: T \in \mathcal{T} \text { and } \operatorname{length}(\partial T \cap \partial \Omega) \neq 0\},
$$

- the set $\mathcal{F}_{T}$ of macro-faces associated to a macro-element $T$ :

$$
\mathcal{F}_{T}:=\left\{F \in \mathcal{F}_{\text {int }} \cup \mathcal{F}_{\text {ext }}: \text { length }(F \cap \partial T) \neq 0\right\} .
$$

We construct an infinite dimensional Trefftz space $X_{T}$, which will be instrumental in the construction of the proposed method. It is defined for $T \in \mathcal{T}$ as the set of functions $\boldsymbol{\omega}_{T}$ satisfying

$$
\begin{aligned}
& \boldsymbol{\omega}_{T} \in H(\nabla \times, T), \\
& \nabla \times\left(\frac{1}{\mu_{T}} \nabla \times \boldsymbol{\omega}_{T}\right)-k_{0}^{2} \varepsilon_{T} \boldsymbol{\omega}_{T}=0, \\
& \boldsymbol{\omega}_{T} \times \mathbf{n}_{T} \in L_{t}^{2}(\partial T), \\
& \mathbf{n}_{T} \times\left(\nabla \times \boldsymbol{\omega}_{T}\right) \in L_{t}^{2}(\partial T),
\end{aligned}
$$

where $\mu_{T}=\mu_{r \mid T}, \varepsilon_{T}=\varepsilon_{r \mid T}$ and $\mathbf{n}_{T}$ is the outward unit normal to $\partial T$.

Remark 2 If $(12 \mathrm{a})$ and $(12 \mathrm{~b})$ are both satisfied, then either $(12 \mathrm{c})$ implies $\sqrt{12 \mathrm{~d}})$ or $(12 \mathrm{~d})$ implies $(12 \mathrm{c})$. In two dimensions, this result is a consequence of the regularity theory of Helmholtz equation which can be found in 29].

It also leads to the global variational space $X_{\mathcal{T}}$ defined element by element,

$$
X_{\mathcal{T}}:=\prod_{T \in \mathcal{T}} X_{T}
$$

Remark 3 Any element $\boldsymbol{\omega}=\left(\boldsymbol{\omega}_{T}\right)_{T \in \mathcal{T}} \in X_{\mathcal{T}}$ is a vector with components $\boldsymbol{\omega}_{T}$ in $L^{2}(T)$. However, $\boldsymbol{\omega}$ can also be identified to a complex valued function of $\Omega$ whose restriction to $T$ is equal to $\boldsymbol{\omega}_{T}$. As a function of $\Omega, \boldsymbol{\omega} \in X_{\mathcal{T}}$ is discontinuous across faces and does not satisfy Maxwell equation in the whole domain $\Omega$ but only in every element $T \in \mathcal{T}$. 


\subsection{The Trefftz continuous formulation}

The Trefftz variational formulation of (5) is deduced from the following duality formula

$$
\int_{\partial T}\left(\mathbf{E}_{T} \cdot \mathbf{n}_{T} \times\left(\frac{1}{\mu_{T}} \nabla \times \overline{\boldsymbol{\omega}_{T}}\right)-\mathbf{n}_{T} \times\left(\frac{1}{\mu_{T}} \nabla \times \mathbf{E}_{T}\right) \cdot \overline{\boldsymbol{\omega}_{T}}\right) d s=0, \text { for } \boldsymbol{\omega}_{T} \in X_{T}
$$

where $\mathbf{E}_{T}$ is the restriction to $T$ of the analytical solution $\mathbf{E}$ to the Maxwell equations (5).

Indeed, since $\mathbf{E}_{T}$ satisfies the Maxwell equation in every element we apply the following Green formula:

$$
\int_{T}(\left(\frac{1}{\mu_{T}} \nabla \times \mathbf{E}_{T}\right) \cdot\left(\nabla \times \overline{\boldsymbol{\omega}_{T}}\right)-\underbrace{\nabla \times\left(\frac{1}{\mu_{T}} \nabla \times \mathbf{E}_{T}\right)}_{k_{0}^{2} \varepsilon_{T} \mathbf{E}_{T}} \cdot \overline{\boldsymbol{\omega}_{T}}) d x=\int_{\partial T}\left(\mathbf{n}_{T} \times\left(\frac{1}{\mu_{T}} \nabla \times \mathbf{E}_{T}\right) \cdot \overline{\boldsymbol{\omega}_{T}}\right) d s .
$$

The test function $\boldsymbol{\omega}$ also satisfies the Maxwell equation in every element. Therefore, by interchanging the roles of $\mathbf{E}$ and $\boldsymbol{\omega}$, we get

$$
\sum_{T \in \mathcal{T}} \int_{T}\left(\left(\frac{1}{\mu_{T}} \nabla \times \mathbf{E}_{T}\right) \cdot\left(\nabla \times \overline{\boldsymbol{\omega}_{T}}\right)-k_{0}^{2} \varepsilon_{T} \mathbf{E}_{T} \cdot \overline{\boldsymbol{\omega}_{T}}\right) d x=\sum_{T \in \mathcal{T}} \int_{\partial T}\left(\mathbf{E}_{T} \cdot \mathbf{n}_{T} \times\left(\frac{1}{\mu_{T}} \nabla \times \overline{\boldsymbol{\omega}_{T}}\right)\right) d s
$$

It remains to subtract $15 \mathrm{~b}$ to $15 \mathrm{a}$ to get 14 ).

The expression on the left hand side of (14) defines a sesquilinear form. The integral over $\partial T$ will be decomposed following the set of faces $\mathcal{F}_{T}$, see $(11)$, of the element $T$

$$
\partial T=\bigcup_{F \in \mathcal{F}_{T}} F
$$

It leads to: $\forall \boldsymbol{\omega} \in X_{\mathcal{T}}$

$$
\widetilde{a}(\mathbf{E}, \boldsymbol{\omega})=\sum_{T \in \mathcal{T}} \sum_{F \in \mathcal{F}_{T}} \widetilde{a}_{T, F}(\mathbf{E}, \boldsymbol{\omega})=0
$$

with

$$
\widetilde{a}_{T, F}(\mathbf{E}, \boldsymbol{\omega}):=\int_{F}\left(\mathbf{E}_{T} \cdot \mathbf{n}_{T} \times\left(\frac{1}{\mu_{T}} \nabla \times \overline{\boldsymbol{\omega}_{T}}\right)-\mathbf{n}_{T} \times\left(\frac{1}{\mu_{T}} \nabla \times \mathbf{E}_{T}\right) \cdot \overline{\boldsymbol{\omega}_{T}}\right) d s
$$

where the solution $\mathbf{E}$ is assumed to belong to the space $X_{\mathcal{T}}$ and $\boldsymbol{\omega}$ to $H(\nabla \times, T)$, see $12 \mathrm{a}$.

We should distinguish the case of an interior face belonging to the interior skeleton $\mathcal{F}_{\text {int }}$ to the case of an exterior face belonging to $\mathcal{F}_{\text {ext }}$.

- If $F \in \mathcal{F}_{\text {int }}$, this face is shared with another element $K$, see Figure 2 , Taking into account the continuity of the solution across the interface $F$

$$
\mathbf{n}_{T} \times\left(\mathbf{n}_{T} \times \mathbf{E}_{T}\right)=\mathbf{n}_{K} \times\left(\mathbf{n}_{K} \times \mathbf{E}_{K}\right) \quad \text { and } \quad \mathbf{n}_{T} \times\left(\frac{1}{\mu_{T}} \nabla \times \mathbf{E}_{T}\right)+\mathbf{n}_{K} \times\left(\frac{1}{\mu_{K}} \nabla \times \mathbf{E}_{K}\right)=0,
$$

we have, for $F \in \mathcal{F}_{\text {int }}$,

$$
\widetilde{a}_{T, F}(\mathbf{E}, \boldsymbol{\omega})=\hat{a}_{T, F}(\mathbf{E}, \boldsymbol{\omega})
$$

with $\hat{a}_{T, F}: X_{\mathcal{T}} \times X_{\mathcal{T}} \longrightarrow \mathbb{C}$ defined by

$$
\hat{a}_{T, F}(\mathbf{E}, \boldsymbol{\omega}):=\int_{F}\left(\mathbf{E}_{K} \cdot \mathbf{n}_{T} \times\left(\frac{1}{\mu_{T}} \nabla \times \overline{\boldsymbol{\omega}_{T}}\right)+\mathbf{n}_{K} \times\left(\frac{1}{\mu_{K}} \nabla \times \mathbf{E}_{K}\right) \cdot \overline{\boldsymbol{\omega}_{T}}\right) d s .
$$

- If $F \in \mathcal{F}_{\text {ext }}$, this face is subject to the impedance condition $\sqrt[5 \mathrm{~b}]{ }$. The form $\widetilde{a}_{T, F}$ is rewritten in a way that the symmetry of the final sesquilinear form is respected

$$
\begin{array}{r}
\widetilde{a}_{T, F}(\mathbf{E}, \boldsymbol{\omega})=\int_{F}\left(\mathbf{E}_{T} \cdot \mathbf{n}_{T} \times\left(\frac{1}{\mu_{T}} \nabla \times \overline{\boldsymbol{\omega}_{T}}\right)+\mathbf{n}_{T} \times\left(\frac{1}{\mu_{T}} \nabla \times \mathbf{E}_{T}\right) \cdot \overline{\boldsymbol{\omega}_{T}}\right) d s \\
-2 \int_{F}(\underbrace{\left(\mathbf{n}_{T} \times\left(\frac{1}{\mu_{T}} \nabla \times \mathbf{E}_{T}\right)\right.}_{i k_{0} Y \mathbf{n}_{T} \times\left(\mathbf{E}_{T} \times \mathbf{n}_{T}\right)-\mathbf{h}} \cdot \overline{\boldsymbol{\omega}_{T}}) d s .
\end{array}
$$

We have, for $F \in \mathcal{F}_{\text {ext }}$,

$$
\widetilde{a}_{T, F}(\mathbf{E}, \boldsymbol{\omega})=\hat{a}_{T, F}(\mathbf{E}, \boldsymbol{\omega})-\hat{\ell}_{T, F}(\boldsymbol{\omega})
$$


with $\hat{a}_{T, F}: X_{\mathcal{T}} \times X_{\mathcal{T}} \longrightarrow \mathbb{C}$ and $\hat{\ell}_{T, F}: X_{\mathcal{T}} \longrightarrow \mathbb{C}$ defined by

$$
\begin{aligned}
\hat{a}_{T, F}(\mathbf{E}, \boldsymbol{\omega}):=\int_{F}\left(\mathbf{E}_{T} \cdot \mathbf{n}_{T} \times\left(\frac{1}{\mu_{T}} \nabla \times \overline{\boldsymbol{\omega}_{T}}\right)+\overline{\boldsymbol{\omega}_{T}} \cdot \mathbf{n}_{T} \times\left(\frac{1}{\mu_{T}} \nabla \times \mathbf{E}_{T}\right)\right) d s & \\
& -2 i k_{0} \int_{F} Y\left(\mathbf{E}_{T} \times \mathbf{n}_{T}\right) \cdot\left(\overline{\boldsymbol{\omega}_{T}} \times \mathbf{n}_{T}\right) d s
\end{aligned}
$$

and

$$
\hat{\ell}_{T, F}(\boldsymbol{\omega})=-2 \int_{F} \mathbf{h} \cdot \overline{\boldsymbol{\omega}_{T}} d s .
$$

Finally, with $\hat{a}_{T, F}$ defined by 20 and $22, \hat{\ell}_{T, F}$ defined by 23 for boundary faces and by taking the convention: $\hat{\ell}_{T, F}(\boldsymbol{\omega})=0, \forall F \in \mathcal{F}_{\text {int }}$, the Trefftz formulation takes the form: find $\mathbf{E} \in X_{\mathcal{T}}$ such that for all $\omega \in X_{\mathcal{T}}$

$$
\sum_{T \in \mathcal{T}} \sum_{F \in \mathcal{F}_{T}} \hat{a}_{T, F}(\mathbf{E}, \boldsymbol{\omega})=\sum_{T \in \mathcal{T}} \sum_{F \in \mathcal{F}_{T}} \hat{\ell}_{T, F}(\boldsymbol{\omega}) .
$$

Remark 4 It is rather natural to add penalization terms to this formulation like in interior penalty DG method (see for example [23]). It is surprising that these additional terms do not lead to any accuracy improvement and deteriorate the linear system conditioning. We refer to [19] for an elaborate theoretical discussion of such penalization in Trefftz formulations.

\section{Discretization of the Trefftz formulation}

We have now established the variational formulation. The next step consists in approximating the space $X_{\mathcal{T}}$. We are first pointing out the existence of an isomorphism between $X_{\mathcal{T}}$ and $L_{t}^{2}(\partial \mathcal{T})$, a trace space defined on the skeleton of the mesh. A Galerkin approximation then leads to a discretization of $L_{t}^{2}(\partial \mathcal{T})$. Many methods exist to choose associated basis functions. For example plane waves or Bessel functions are analytical local solutions on Trefftz elements and can be employed as basis functions. A comparative discussion of plane waves and Bessel functions discretizations can be found in [16].

In this article, a different approach is proposed. Local basis functions are defined as solutions of a boundary value problem (25). An impedance boundary condition is considered to design a well-posed problem. Perfect conductor condition could have been as well considered but resonances phenomena could occur. Once those Trefftz basis functions are defined, (24) can not be computed analytically, since the solution operator associated to the isomorphism between $L_{t}^{2}(\partial \mathcal{T})$ and $X_{\mathcal{T}}$ is not explicit. To overcome this difficulty, we propose to use a second approximation based on a FEM. In [5, 21, 22, BEM is proposed to compute the local basis functions for Helmholtz equations. However, FEM are, to our opinion, more flexible and can deal with a wider variety of physical models.

\subsection{Identification of spaces $X_{T}$ and $X_{\mathcal{T}}$ with $L_{t}^{2}(\partial T)$ and $L_{t}^{2}(\partial \mathcal{T})$}

We start with the identification of the local space $X_{T}$ with $L_{t}^{2}(\partial T)$ for each $T \in \mathcal{T}$. It is based on the following result: let $\mathbf{g}_{T} \in L_{t}^{2}(\partial T)$ and $Y_{T} \in L^{\infty}(\partial T)$ with a strictly non-positive real part, then there exists a unique $\boldsymbol{\omega}_{T} \in H(\nabla \times, T)$ (see [32]) such that

$$
\begin{aligned}
& \nabla \times\left(\frac{1}{\mu_{T}} \nabla \times \boldsymbol{\omega}_{T}\right)-k_{0}^{2} \varepsilon_{T} \boldsymbol{\omega}_{T}=0 \text { in } \quad T, \\
& i k_{0} Y_{T} \mathbf{n}_{T} \times\left(\boldsymbol{\omega}_{T} \times \mathbf{n}_{T}\right)-\mathbf{n}_{T} \times\left(\frac{1}{\mu_{T}} \nabla \times \boldsymbol{\omega}_{T}\right)=\mathbf{g}_{T} \quad \text { on } \quad \partial T,
\end{aligned}
$$

where we recall that physical parameters are constant in the element $T$. The solution of (25) satisfies the regularity statements $(12 \mathrm{c})$ and $(12 \mathrm{~d})$. Consequently, $\boldsymbol{\omega}_{T}$ belongs to the space $X_{T}$. Reciprocally all functions of $X_{T}$ are obviously solutions of the problem 25 . In particular, the local solution operator $\mathbf{S}_{T}: L_{t}^{2}(\partial T) \longrightarrow X_{T}$ defined by

$$
\mathbf{S}_{T} \mathbf{g}_{T}=\boldsymbol{\omega}_{T}
$$

where $\boldsymbol{\omega}_{T}$ is the solution of 25$)$, induces an isomorphism between the spaces $L_{t}^{2}(\partial T)$ and $X_{T}$. This leads to the following characterization of the space $X_{T}$ :

$$
X_{T}=\left\{\mathbf{S}_{T} \mathbf{g}_{T} \text { such that } \mathbf{g}_{T} \in L_{t}^{2}(\partial T)\right\} .
$$


Remark 5 At most frequencies, it would have been also possible to consider Perfect Electric Condition (PEC) or alternatively Perfect Magnetic Condition (PMC) to parametrize $X_{T}$. In this case 25b would have been replaced by one of the following boundary conditions

$$
\begin{aligned}
& \mathbf{n}_{T} \times\left(\boldsymbol{\omega}_{T} \times \mathbf{n}_{T}\right)=\mathbf{g}_{T} \quad \text { on } \quad \partial T, \\
& \mathbf{n}_{T} \times\left(\frac{1}{\mu_{T}} \nabla \times \boldsymbol{\omega}_{T}\right)=\mathbf{g}_{T} \quad \text { on } \quad \partial T .
\end{aligned}
$$

However, the PEC and PMC are associated to spurious modes predicted by the Maxwell spectral theory in bounded domains. These resonances phenomena do not occur with an impedance boundary condition.

The global Trefftz space $X_{\mathcal{T}}$, see $(13)$, is therefore in bijection with the space

$$
L_{t}^{2}(\partial \mathcal{T}):=\prod_{T \in \mathcal{T}} L_{t}^{2}(\partial T)
$$

through the global solution operator $\mathbf{S}: L_{t}^{2}(\partial \mathcal{T}) \longrightarrow X_{\mathcal{T}}$. We associate to the solution $\mathbf{E}$ (resp. the test functions $\boldsymbol{\omega})$ an element of the trace space $\mathbf{f}($ resp. $\mathbf{g})$ by $\mathbf{S f}=\mathbf{E}($ resp. $\mathbf{S g}=\boldsymbol{\omega})$ which is defined by 26 on every element by $\mathbf{E}_{T}=\mathbf{S}_{T} \mathbf{f}_{T}$ (resp. $\boldsymbol{\omega}_{T}=\mathbf{S}_{T} \mathbf{g}_{T}$ ), see Fig. 3 .

The Trefftz formulation $(24)$ can be written in the following form: find $\mathbf{f} \in L_{t}^{2}(\partial \mathcal{T})$ such that for all $\mathbf{g} \in L_{t}^{2}(\partial \mathcal{T})$

$$
\sum_{T \in \mathcal{T}} \sum_{F \in \mathcal{F}_{T}} \hat{a}_{T, F}(\mathbf{S f}, \mathbf{S g})=\sum_{T \in \mathcal{T}} \sum_{F \in \mathcal{F}_{T}} \hat{\ell}_{T, F}(\mathbf{S g}) .
$$

The variational problem $(29)$ involves the tangential trace of the rotational. Most of numerical methods are not adapted to the evaluation of this quantity. An important feature of the presented numerical method consists in computing the rotational thanks to $25 \mathrm{~b}$ that is satisfied by $\boldsymbol{\omega}=\mathbf{S g}$. Similarly, $\mathbf{E}=\mathbf{S f}$ satisfies the following equation:

$$
\mathbf{n}_{T} \times\left(\frac{1}{\mu_{T}} \nabla \times \mathbf{S}_{T} \mathbf{f}_{T}\right)=i k_{0} Y_{T} \mathbf{n}_{T} \times\left(\mathbf{S}_{T} \mathbf{f}_{T} \times \mathbf{n}_{T}\right)-\mathbf{f}_{T}
$$

Finally, we have the variational problem: find $\mathbf{f} \in L^{2}(\partial \mathcal{T})$ such that for all $\mathbf{g} \in L^{2}(\partial \mathcal{T})$,

$$
\sum_{T \in \mathcal{T}} \sum_{F \in \mathcal{F}_{T}} a_{T, F}(\mathbf{f}, \mathbf{g})=\sum_{T \in \mathcal{T}} \sum_{F \in \mathcal{F}_{T}} \ell_{T, F}(\mathbf{g}),
$$

where $a_{T, F}: L_{t}^{2}(\partial \mathcal{T}) \times L_{t}^{2}(\partial \mathcal{T}) \rightarrow \mathbb{C}$ and $\ell_{T, F}: L_{t}^{2}(\partial \mathcal{T}) \rightarrow \mathbb{C}$, defined by

$$
\begin{aligned}
& a_{T, F}(\mathbf{f}, \mathbf{g}):=\hat{a}_{T, F}(\mathbf{S f}, \mathbf{S g})=i k_{0} \int_{F}\left(\overline{Y_{T}}-Y_{K}\right)\left(\mathbf{n}_{K} \times \mathbf{S}_{K} \mathbf{f}_{K}\right) \cdot\left(\mathbf{n}_{T} \times \overline{\mathbf{S}_{T} \mathbf{g}_{T}}\right) d s \\
& -\int_{F}\left(\overline{\mathbf{g}_{T}} \cdot \mathbf{S}_{K} \mathbf{f}_{K}+\mathbf{f}_{K} \cdot \overline{\mathbf{S}_{T} \mathbf{g}_{T}}\right) d s \quad \text { for } F \in \mathcal{F}_{i n t}, \\
& a_{T, F}(\mathbf{f}, \mathbf{g}):=\hat{a}_{T, F}(\mathbf{S f}, \mathbf{S g})=i k_{0} \int_{F}\left(Y_{T}+\overline{Y_{T}}-2 Y\right)\left(\mathbf{n}_{T} \times \mathbf{S}_{T} \mathbf{f}_{T}\right) \cdot\left(\mathbf{n}_{T} \times \overline{\mathbf{S}_{T} \mathbf{g}_{T}}\right) d s \\
& -\int_{F}\left(\overline{\mathbf{g}_{T}} \cdot \mathbf{S}_{T} \mathbf{f}_{T}+\mathbf{f}_{T} \cdot \overline{\mathbf{S}_{T} \mathbf{g}_{T}}\right) d s \quad \text { for } F \in \mathcal{F}_{\text {ext }}, \\
& \ell_{T, F}(\mathbf{g}):=\hat{\ell}_{T, F}(\mathbf{S g})=0 \quad \text { for } F \in \mathcal{F}_{i n t}, \\
& \ell_{T, F}(\mathbf{g}):=\hat{\ell}_{T, F}(\mathbf{S g})=-2 \int_{F} \mathbf{h} \cdot \overline{\mathbf{S}_{T} \mathbf{g}_{T}} d s \quad \text { for } F \in \mathcal{F}_{\text {ext }} .
\end{aligned}
$$

Remark 6 Trefftz methods lead to variational formulations based on trace spaces. The construction of (31) supports this particularity.

Remark 7 In case $Y_{T}=Y_{K}=Y \in \mathbb{R}$, the formulation is drastically simplified for both cases $F \in F_{\text {int }}$ and $F \in F_{\text {ext }}$.

Remark 8 Considering a complex bilinear form instead of a sesquilinear form will lead to an alternative interesting simpler formulation. Indeed, the problem $\sqrt{31}$ will be simplified by erasing the complex conjugate symbol. Both formulations should be numerically tested to determine advantages and disadvantages of the two formulations. Both formulations are equivalent when $Y_{T}$ is real. In the case $Y_{T}=Y_{K}=Y \in \mathbb{C}$, this second formulation can also be drastically simplified. 


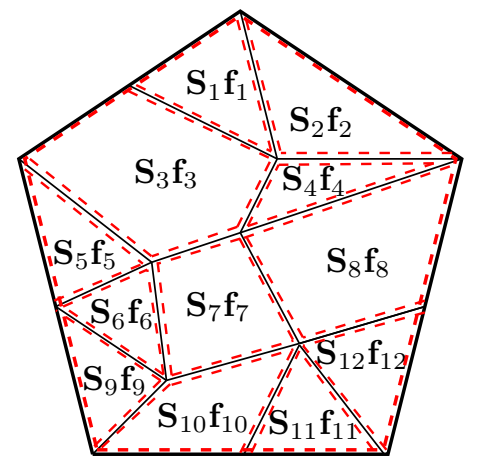

Fig. 3 Decomposition of the solution with the global solution operator $\mathbf{S}$ element by element.

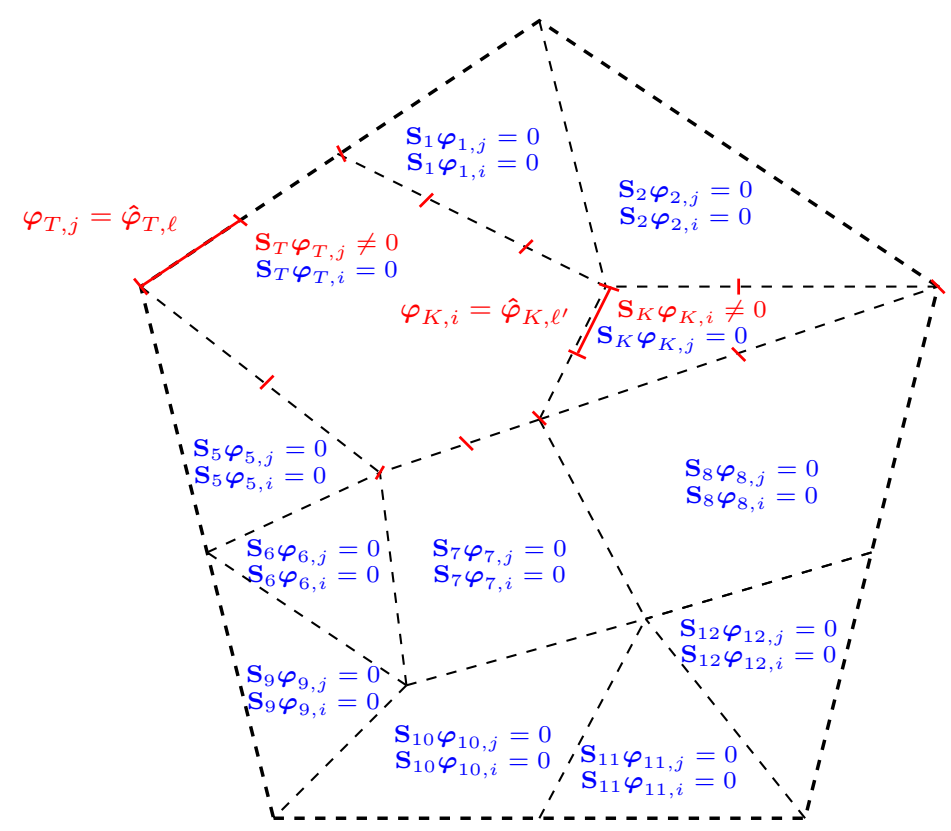

Fig. 4 Interaction between two local basis functions $\varphi_{T, i}$ and $\varphi_{K, j}$, between two neighboring elements, where $j=\operatorname{loc} 2 g \operatorname{lob}(T, \ell)$ and $i=\operatorname{loc} 2 g l o b\left(K, \ell^{\prime}\right)$.

\subsection{Galerkin approximation of the Trefftz formulation}

The discretization of (31) goes through the choice of a finite dimensional space to approximate $L_{t}^{2}(\partial \mathcal{T})$. This space is constructed from a partition $\mathcal{F}_{F, \mu}$ in segments of each macro-face $F \in \mathcal{F}_{\text {int }} \cup \mathcal{F}_{\text {ext }}$. They will be called micro-faces.

From these partitions, we then define for each $T \in \mathcal{T}$, the set $\mathcal{F}_{T, \mu}:=\bigcup_{F \in \mathcal{F}_{T}} \mathcal{F}_{F, \mu}$ of micro-faces associated to $T$ and an approximation space of $L_{t}^{2}(\partial T)$ :

$$
V_{T, \mu}^{q}:=\left\{\mathbf{v}: \partial T \rightarrow \mathbb{C}^{2}: \forall F_{\mu} \in \mathcal{F}_{T, \mu}, \mathbf{v}_{\mid F_{\mu}} \in\left[\mathcal{P}^{q}\left(F_{\mu}\right)\right]^{2} \text { such that } \mathbf{v}_{\mid F_{\mu}} \cdot \mathbf{n}_{T}=0\right\},
$$

where $\mathcal{P}^{q}\left(F_{\mu}\right):=\left\{p: F_{\mu} \rightarrow \mathbb{C}: p \circ \Phi_{F_{\mu}} \in \mathcal{P}^{q}([0,1])\right\}$ with $\Phi_{F_{\mu}}$ being the affine mapping from $[0,1]$ to $F_{\mu}$ and $\mathcal{P}^{q}([0,1])$ is the space of polynomials of degree $q \in \mathbb{N}$. An alternative definition can be found in [32], that is : $\mathcal{P}^{q}\left(F_{\mu}\right)$ is the space of polynomials of maximum degree $q \in \mathbb{N}$ in arc length on $F_{\mu}$.

Finally a conforming approximation space of $L_{t}^{2}(\partial \mathcal{T})$ is defined by

$$
V_{\mu}^{q}:=\prod_{T \in \mathcal{T}} V_{T, \mu}^{q}
$$

We propose a first discrete Trefftz formulation of $(31)$ as follows: find $\mathbf{f}_{\mu}^{q} \in V_{\mu}^{q}$ such that for all $\mathbf{g}_{\mu}^{q} \in V_{\mu}^{q}$,

$$
a\left(\mathbf{f}_{\mu}^{q}, \mathbf{g}_{\mu}^{q}\right)=\ell\left(\mathbf{g}_{\mu}^{q}\right),
$$

where

$$
a\left(\mathbf{f}_{\mu}^{q}, \mathbf{g}_{\mu}^{q}\right):=\sum_{T \in \mathcal{T}} \sum_{F \in \mathcal{F}_{T}} a_{T, F}\left(\mathbf{f}_{\mu}^{q}, \mathbf{g}_{\mu}^{q}\right) \text { and } \ell\left(\mathbf{g}_{\mu}^{q}\right):=\sum_{T \in \mathcal{T}} \sum_{F \in \mathcal{F}_{T}} \ell_{T, F}\left(\mathbf{g}_{\mu}^{q}\right)
$$

A linear system is then associated to this variational formulation by introducing a basis of $V_{\mu}^{q}$. As for any discontinuous finite element, we refer to local and global basis functions.

- Local basis functions are defined on $\partial T$ and are denoted by $\hat{\varphi}_{T, \ell} \in V_{T, \mu}^{q}$ with $1 \leq \ell \leq(q+1) N_{T, \mu}$, where $N_{T, \mu}$ is the number of micro-faces of $T$ and $(q+1)$ is the dimension of the local polynomial approximation space.

- Global basis vectors can be seen as global "functions". They are defined on $\partial \mathcal{T}$ by $\varphi_{i} \in V_{\mu}^{q}$ with $1 \leq i \leq$ $\operatorname{card}\left(V_{\mu}^{q}\right)$. The dimension of the space $V_{\mu}^{q}$ is given by

$$
\operatorname{card}\left(V_{\mu}^{q}\right)=\sum_{T \in \mathcal{T}}(q+1) N_{T, \mu}
$$


Local (resp. global) basis functions are defined on the boundary $\partial T$ (resp. $\partial \mathcal{T}$ ) and lead to local functions $\boldsymbol{\omega}_{T}=\mathbf{S}_{T} \boldsymbol{\varphi}_{T}$ (resp. global functions $\boldsymbol{\omega}=\mathbf{S} \boldsymbol{\varphi}$ ) defined on the whole element $T$ (resp. the whole set $\mathcal{T}$ ).

To construct matrices associated to (34), we must define a link between $\hat{\varphi}_{T, \ell}$ and $\varphi_{i}$. That is why we introduce a local numbering and a global numbering linked by the following bijective operator loc2glob:

$$
i=\operatorname{loc} 2 \operatorname{glob}(T, \ell)
$$

where $i$ is the global number associated to the local number $\ell=1, \ldots,(q+1) N_{T, \mu}$ of the macro-element $T \in \mathcal{T}$.

The global basis functions $\left(\boldsymbol{\varphi}_{i}\right)_{i=1, \ldots, \operatorname{card}\left(V_{\mu}^{q}\right)}$ are constructed as follows: if $i=\operatorname{loc} 2 \operatorname{glob}(T, \ell)$ then $\boldsymbol{\varphi}_{i}:=$ $\left(\varphi_{K, i}\right)_{K \in \mathcal{T}}$ are defined by

$$
\begin{cases}\boldsymbol{\varphi}_{K, i}=0, & \text { if } K \neq T \\ \varphi_{K, i}=\hat{\varphi}_{T, \ell}, & \text { if } K=T\end{cases}
$$

The basis defined above leads to a characterization of the space $V_{\mu}^{q}$. Indeed, we can describe $\mathbf{f}_{\mu}^{q}=\left(\mathbf{f}_{T, \mu}^{q}\right)_{T \in \mathcal{T}} \in$ $V_{\mu}^{q}$ using $\varphi_{i}$ :

$$
\mathbf{f}_{\mu}^{q}=\sum_{j=1}^{\operatorname{card}\left(V_{\mu}^{q}\right)} f_{j} \boldsymbol{\varphi}_{j}, \text { with } f_{j} \in \mathbb{C} .
$$

Let us consider the restriction $\mathbf{f}_{T, \mu}^{q}$ of $\mathbf{f}_{\mu}^{q}$ on $T \in \mathcal{T}$,

$$
\mathbf{f}_{T, \mu}^{q}=\sum_{j=1}^{\operatorname{card}\left(V_{\mu}^{q}\right)} f_{j} \boldsymbol{\varphi}_{T, j}
$$

where $\boldsymbol{\varphi}_{T, j}$ is the restriction of $\boldsymbol{\varphi}_{j}$ on $T$. According to (38), only components from the element $T$ are non-zero, see Fig. 4. This sum can thus be formulated for local basis functions of $T$ only:

$$
\mathbf{f}_{T, \mu}^{q}=\sum_{\ell=1}^{(q+1) N_{T, \mu}} f_{\operatorname{loc} 2 \operatorname{glob}(T, \ell)} \hat{\boldsymbol{\varphi}}_{T, \ell}
$$

Finally, the formulation (34) can be rewritten as follows: find $\left(f_{j}\right)_{j=1, \ldots, \operatorname{card}\left(V_{\mu}\right)}$ such that $\forall i \in 1, \ldots, \operatorname{card}\left(V_{\mu}\right)$,

$$
\sum_{j=1}^{\operatorname{card}\left(V_{\mu}\right)} f_{j} a\left(\boldsymbol{\varphi}_{j}, \boldsymbol{\varphi}_{i}\right)=\ell\left(\boldsymbol{\varphi}_{i}\right) .
$$

If $j=\operatorname{loc} 2 \operatorname{glob}(T, \ell)$ and $i=\operatorname{loc} 2 \operatorname{glob}\left(K, \ell^{\prime}\right)$ then $a\left(\boldsymbol{\varphi}_{j}, \boldsymbol{\varphi}_{i}\right)=0$ if, and only if,

$$
(T \neq K \quad \text { and length }(\partial T \cap \partial K) \neq 0) \quad \text { or } \quad(T=K \text { s.t length }(\partial \Omega \cap \partial T)=0),
$$

where length is defined in (9).

Therefore the matrix associated to the sesquilinear form $a$ is sparse.

The linear system (42) can then be used to find $\mathbf{f}_{\mu}^{q} \in V_{\mu}^{q}$. To solve this system we must compute $\mathbf{S} \boldsymbol{\varphi}_{i}$ (see (31b) and (31c)). However, $\mathbf{S}$ is usually not known, such that $\boldsymbol{\omega}=\mathbf{S} \boldsymbol{\varphi}_{i} \in X_{T}, i=1, \ldots,(q+1) N_{T, \mu}$, cannot be known explicitly. We have to determine an approximation $\mathbf{S}_{\nu}$ of the linear operator $\mathbf{S}$. The latter can be described by using several methods as a FDM, an integral equations method, or a FEM. In this paper, Nédélec finite elements [32] are taken as an example and are described in the next section.

Remark 9 For this reason our Trefftz method should be called quasi-Trefftz. Indeed, effective basis functions are not exact solutions of our Maxwell problem. 

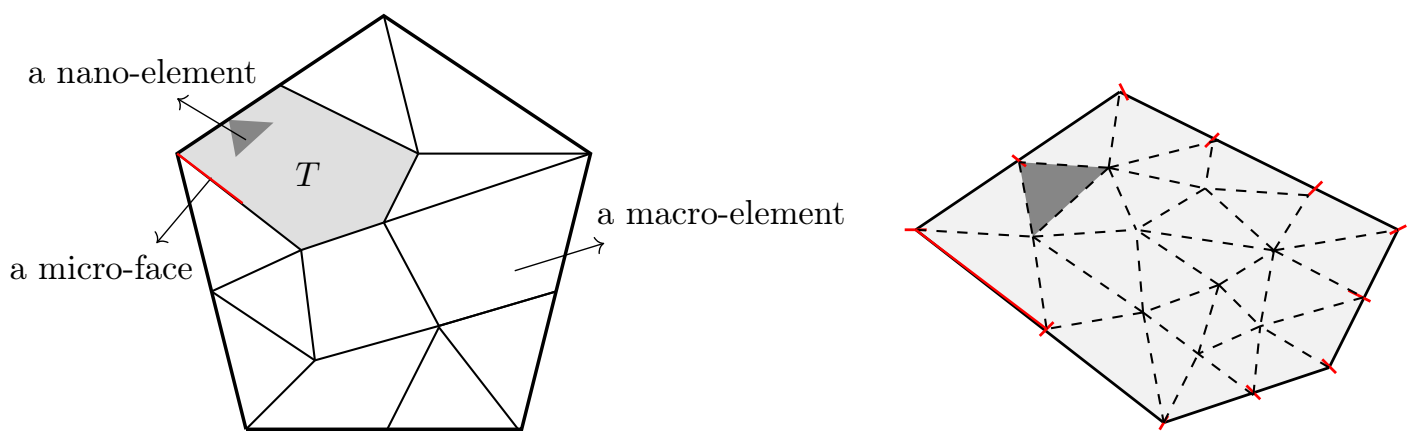

Fig. 5 Discretization of a macro-element $T$.

\subsection{Example of finite element approximation of $\mathbf{S}$}

The construction of the Trefftz linear system requires to compute an approximation of $\mathbf{S} \boldsymbol{\varphi}_{j}=\left(\mathbf{S}_{K} \boldsymbol{\varphi}_{K, j}\right)_{K \in \mathcal{T}}$. The function $\mathbf{S}_{K} \boldsymbol{\varphi}_{K, j}$ is equal to zero except on the macro-element $K=T$ such that $j=\operatorname{loc} 2 \mathrm{glob}(T, \ell)$. In this element, an approximation of $\mathbf{S}_{T} \boldsymbol{\varphi}_{j}$ is obtained by discretizing the local problem 25] for $\mathbf{g}_{T}=\hat{\boldsymbol{\varphi}}_{T, \ell}$. This problem reads: find $\hat{\boldsymbol{\omega}}_{T, \ell}=\mathbf{S}_{T} \boldsymbol{\varphi}_{T, j} \in H(\nabla \times, T)$, such that

$$
\begin{aligned}
\nabla \times\left(\frac{1}{\mu_{T}} \nabla \times \hat{\boldsymbol{\omega}}_{T, \ell}\right)-k_{0}^{2} \varepsilon_{T} \hat{\boldsymbol{\omega}}_{T, \ell}=0 \quad \text { in } \quad T, \\
i k_{0} Y_{T} \mathbf{n}_{T} \times\left(\hat{\boldsymbol{\omega}}_{T, \ell} \times \mathbf{n}_{T}\right)-\mathbf{n}_{T} \times\left(\frac{1}{\mu_{T}} \nabla \times \hat{\boldsymbol{\omega}}_{T, \ell}\right)=\hat{\boldsymbol{\varphi}}_{T, \ell} \quad \text { on } \quad \partial T .
\end{aligned}
$$

It will be approximated with a high-order Nédélec FEM, see for example [34, 35]. The associated variational formulation of t44 takes the form: find $\hat{\boldsymbol{\omega}}_{T, \ell}=\mathbf{S}_{T} \boldsymbol{\varphi}_{T, j} \in H(\nabla \times, T)$ such that $\forall \boldsymbol{\psi} \in H(\nabla \times, T)$

$$
\begin{array}{r}
-k_{0}^{2} \int_{T} \varepsilon_{T}\left(\hat{\boldsymbol{\omega}}_{T, \ell} \cdot \overline{\boldsymbol{\psi}}\right) d x+\int_{T} \frac{1}{\mu_{T}}\left(\nabla \times \hat{\boldsymbol{\omega}}_{T, \ell}\right) \cdot(\nabla \times \overline{\boldsymbol{\psi}}) d x+i k_{0} \int_{\partial T} Y_{T}\left(\mathbf{n}_{T} \times \hat{\boldsymbol{\omega}}_{T, \ell}\right) \cdot\left(\mathbf{n}_{T} \times \overline{\boldsymbol{\psi}}\right) d s \\
=\int_{\partial T}\left(\hat{\boldsymbol{\varphi}}_{T, \ell} \cdot \overline{\boldsymbol{\psi}}\right) d s
\end{array}
$$

Now, let $\mathcal{T}_{\nu}(T)$ be a triangular mesh of the macro-element $T \in \mathcal{T}$, see Fig 5

The Nédélec finite element space $V_{T, \nu}^{p}$ of order $p \in \mathbb{N}$, see [32], is defined by :

$$
V_{T, \nu}^{p}:=\left\{\mathbf{v} \in H(\nabla \times, T): \forall T_{\nu} \in \mathcal{T}_{\nu}(T), \mathbf{v}_{\mid T_{\nu}} \in \mathcal{N}^{p}\left(T_{\nu}\right)\right\}
$$

where

$$
\mathcal{N}^{p}\left(T_{\nu}\right)=\mathcal{P}^{p}\left(T_{\nu}\right)^{2}+\mathcal{S}^{p+1}\left(T_{\nu}\right)
$$

with $\mathcal{S}^{p}\left(T_{\nu}\right)=\left\{\mathbf{p} \in\left(\tilde{\mathcal{P}}^{p}\left(T_{\nu}\right)\right)^{2}:\left(\begin{array}{l}x \\ y\end{array}\right) \cdot \mathbf{p}=0\right\}$ and $\tilde{\mathcal{P}}^{p}\left(T_{\nu}\right)$ the space of homogeneous polynomial functions of degree $p$.

Finally, a finite element approximation $\mathbf{S}_{T, \nu}^{p} \boldsymbol{\varphi}_{T, j}$ of $\mathbf{S}_{T} \boldsymbol{\varphi}_{T, j}$ is computed by solving the following discrete problem: find $\hat{\boldsymbol{\omega}}_{T, \ell}^{\nu, p}=\mathbf{S}_{T, \nu}^{p} \hat{\boldsymbol{\varphi}}_{T, \ell} \in V_{T, \nu}^{p}$, such that $\forall \boldsymbol{\psi} \in V_{T, \nu}^{p}$

$$
\begin{array}{r}
-k_{0}^{2} \int_{T} \varepsilon_{T}\left(\hat{\boldsymbol{\omega}}_{T, \ell}^{\nu, p} \cdot \overline{\boldsymbol{\psi}}\right) d x+\int_{T} \frac{1}{\mu_{T}}\left(\nabla \times \hat{\boldsymbol{\omega}}_{T, \ell}^{\nu, p}\right) \cdot(\nabla \times \overline{\boldsymbol{\psi}}) d x+i k_{0} \int_{\partial T} Y_{T}\left(\mathbf{n}_{T} \times \hat{\boldsymbol{\omega}}_{T, \ell}^{\nu, p}\right) \cdot\left(\mathbf{n}_{T} \times \overline{\boldsymbol{\psi}}\right) d s \\
=\int_{\partial T}\left(\hat{\boldsymbol{\varphi}}_{T, \ell} \cdot \overline{\boldsymbol{\psi}}\right) d s
\end{array}
$$

The Nédélec FEM is implemented by using basis functions deduced from the ones proposed in [14] for a high-order Raviart-Thomas approximation, by applying a simple $\pi / 2$ rotation. 


\section{Numerical investigation of the proposed Trefftz method}

This section presents some numerical results from the implementation of the Trefftz method. The first part focuses on a numerical analysis of the convergence and the pollution error. The second part is qualitative and deals with classical examples.

Our method is natively adapted to small or large macro-elements since the parametrization of the space $X_{T}$ by an impedance condition avoids spurious modes, see Remark 5. In this paper, we subdivide the computation domain into macro-elements whose sizes are at most a few wavelengths. This size is a good compromise in terms of computational effort. Indeed, it induces an interesting sparsity of the Trefftz linear system, and it avoids increases of both the system conditioning and the memory required for the local solver.

Our implementation is based on a particular choice of local basis functions. Indeed, they have distinct local numberings, ranging from 1 to $(q+1) N_{T, \mu}$ (see 3.2), and have Lagrange interpolation polynomial values. They are defined as follows:

- the subscript $\ell$ is defined by $\ell=\left(i_{\mu}-1\right)(q+1)+l_{\mu}$, where $i_{\mu}$ is the micro-face number of $F_{\mu} \in \mathcal{F}_{T, \mu}$ such that $1 \leq i_{\mu} \leq N_{T, \mu}$, and $\ell_{\mu}$ is the local number of the basis function such that $1 \leq \ell_{\mu} \leq q+1$,

- for all $\bar{F}_{\mu} \in \overline{\mathcal{F}}_{T, \mu}$,

$$
\hat{\varphi}_{T, \ell \mid F_{\mu}} \circ \Phi_{F_{\mu}}=\left\{\begin{array}{l}
(0,0)^{T} \text { if } F_{\mu} \neq F_{i_{\mu}} \\
L_{\ell_{\mu}}^{q} \mathbf{t}_{\partial T} \text { if } F_{\mu}=F_{i_{\mu}}
\end{array},\right.
$$

where the unit tangent vector function is defined by $\mathbf{t}_{\partial T}=\mathbf{n}_{T} \times(0,0,1)^{T}$ and $L_{\ell_{\mu}}^{q}$ is the $\ell_{\mu}{ }^{\text {th }}$ Lagrange interpolation polynomial constructed from the $(q+1)$ Gauss points in $[0,1]$.

In our implementation, the mesh $\mathcal{T}_{\nu}(T)$ associated to each macro-element $T$ verifies the following property:

$\forall T_{\nu} \in \mathcal{T}_{\nu}(T), \quad$ either length $\left(\partial T_{\nu} \cap \partial T\right)=0 \quad$ or $\exists F_{\mu} \in \mathcal{F}_{T, \mu}$ such that $\partial T_{\nu} \cap \partial T=F_{\mu}$ (see Fig. 5).

In other words, we don't refine the mesh $\mathcal{T}_{\nu}(T)$ in order to improve the quality of the approximation $S_{T, \nu}^{p}$. Consequently, only p-convergence of Nédélec FEM has yet been implemented. Nevertheless, in presence of singularities, a more efficient approach would be an hp-version of the local solver. A DG method using geometrically graded meshes is especially adapted.

Finally, the local admittance $Y_{T}$ used in the definition of $\mathbf{S}$ (see $(25 \mathrm{~b})$ ) is chosen to be equal to $\sqrt{\varepsilon_{T} / \mu_{T}}$.

4.1 Numerical error analysis

\subsubsection{Relationship between $V_{T, \mu}^{q}$ and $V_{T, \nu}^{p}$}

We study the link between approximation orders $q$ and $p$ by considering the following problem:

$$
\begin{aligned}
& \nabla \times(\nabla \times \mathbf{E})-k_{0}^{2} \mathbf{E}=0 \quad \text { in } \Omega, \\
& i k_{0} \mathbf{n} \times(\mathbf{E} \times \mathbf{n})-\mathbf{n} \times(\nabla \times \mathbf{E})=\mathbf{h}^{i n c} \quad \text { on } \partial \Omega,
\end{aligned}
$$

where $\mathbf{h}^{i n c}=i k_{0} \mathbf{n} \times\left(\mathbf{E}^{i n c} \times \mathbf{n}\right)-\mathbf{n} \times\left(\nabla \times \mathbf{E}^{i n c}\right)$, with $\mathbf{E}^{i n c}$ an incident plane wave whose direction of propagation is $(1,1)^{T}$, and the computational domain $\Omega$ is represented in Fig. 6 .

The analytical solution of this problem is obviously $\mathbf{E}=\mathbf{E}^{i n c}$ due to its well-posedness character.

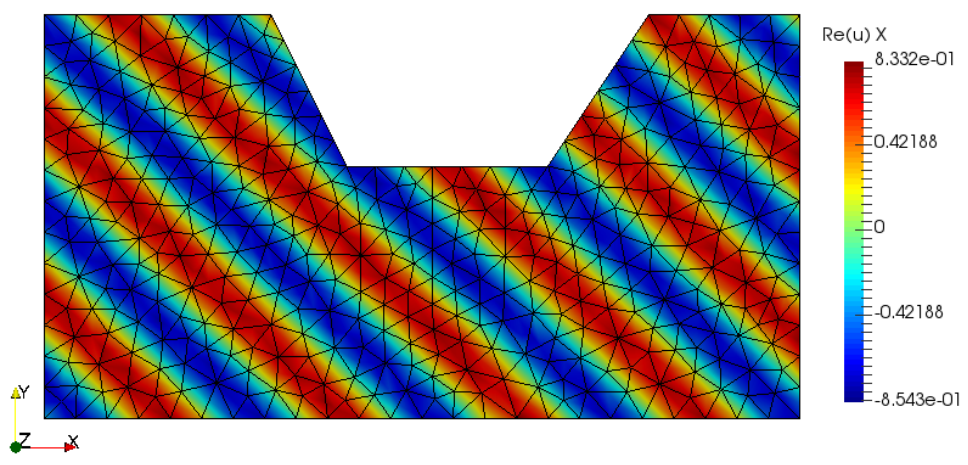

Fig. 6 The macro-element $\Omega$, an example of triangular mesh $\mathcal{T}_{\nu}(\Omega)$ and the representation of the analytical solution. 
For the simulation, only one macro-element is used i.e $\mathcal{T}=\{\Omega\}$. As the example displayed in Fig. $6, \mathcal{T}_{\nu}(\Omega)$ is a triangular mesh. In order to study their relationship, approximation orders $q$ and $p$ are respectively varying from 0 to 2 and from 0 to 4 .

Let us introduce the relative error in H-curl norm:

$$
\mathbf{e}:=\frac{\sqrt{\left\|\mathbf{E}_{\mu, \nu}^{q, p}-\mathbf{E}\right\|_{0, \Omega}^{2}+\left\|\nabla \times\left(\mathbf{E}_{\mu, \nu}^{q, p}-\mathbf{E}\right)\right\|_{0, \Omega}^{2}}}{\sqrt{\|\mathbf{E}\|_{0, \Omega}^{2}+\|\nabla \times \mathbf{E}\|_{0, \Omega}^{2}}},
$$

where $\mathbf{E}_{\mu, \nu}^{q, p}$ denotes the numerical solution of our Trefftz scheme.

Curves obtained from our simulation, see Fig. 7. show the relative error in function of the quantity $k h /(2 \pi(q+$ 1)) which is equivalent to the inverse of the number of points per wavelength. In our case, $h$ is the longest length of micro-faces.
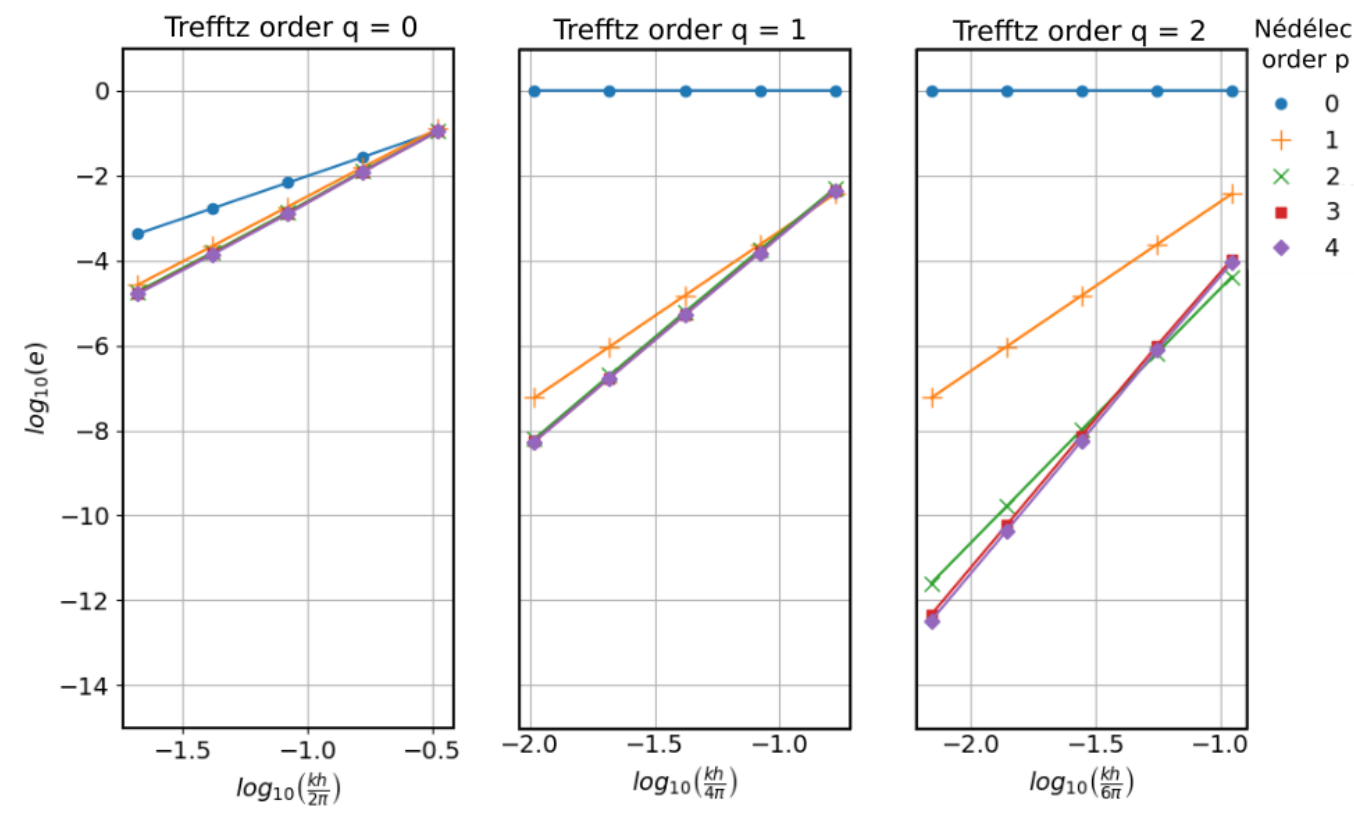

Fig. 7 Convergence curves in H-curl error of the Trefftz scheme for some local Nédélec approximations.

These plots show that the Trefftz order $q$ and the FEM order $p$ must be carefully chosen. Indeed, if $p<q$, a projection of the "trace" basis functions $\varphi_{i}$ onto lower-order polynomials occurs leading to a loss in precision and convergence. Consequently, the condition $p \geq q$ must be satisfied for the proposed Trefftz scheme. As a matter of fact, the higher the local Nédélec FEM order, the closer the basis functions are to the exact Maxwell solutions. When $\mathrm{p}$ is sufficiently large, the lack of conformity in our local approximation becomes then negligible.

Mathematically, the convergence rate of the error induced by the variational crime perpetrated in the discretization of the sesquilinear form should be at least of the same order as the one of the underlying Trefftz method. The bound of this condition, i.e $p=q$, denotes a convergence rate similar to Nédélec FEM of order $p$. In case $p>q$, a super-convergence phenomenon is pointed out. By super-convergence, we mean that the Trefftz method converges at a higher rate than a comparable Nédélec FEM with the same number of degrees of freedom on the boundary. Let us recall that introducing extra degrees of freedom for the Nédélec finite element solver has no extra cost since they are eliminated from the final system. In all plots, highest order curves are close to each other. It reveals a saturation phenomenon. The superposition of curves in Fig. 7 demonstrates that the local solver is almost exact in comparison with the Trefftz solver. It brings the optimal choice $p=q+1$. This is exactly what one should expect from a Trefftz method and motivates their use instead of standard ones.

\subsubsection{Evaluation of the pollution error}

One of the main motivations of Trefftz type methods is reducing the impact of the pollution effect, by which more classical FEM tend to be limited when the computational domain becomes large in terms of wavelength. We analyze the behaviour of the proposed Trefftz scheme regarding this effect by considering the following 
problem:

$$
\begin{array}{rlrl}
\nabla \times \nabla \times \mathbf{E}-k_{0}^{2} \mathbf{E}=0 & & \text { in } \Omega=[0, L] \times[0,1], \\
\mathbf{n} \times(\mathbf{E} \times \mathbf{n}) & =0 & & \text { if } y=0 \text { or } y=1, \\
\mathbf{n} \times(\mathbf{E} \times \mathbf{n}) & =-1 & & \text { if } x=0, \\
i k_{0} \mathbf{n} \times(\mathbf{E} \times \mathbf{n})-\mathbf{n} \times \nabla \times \mathbf{E}=0 & & \text { if } x=L,
\end{array}
$$

where $k_{0}=2 \pi$ and $L=M \lambda_{0}$ with $\lambda_{0}=2 \pi / k_{0}$ and $M=10, \ldots, 200$.

This problem is slightly different than the one initially considered in this paper (see (5)). Indeed, in this latter, we have decided to consider only an impedance boundary condition to simplify the presentation. Dirichlet boundary conditions as these ones used in 52 can straightforwardly be included in the scheme.

The problem $(52$ models a duct of height 1 and length $L$ where an incoming plane wave is generated at $x=0$, propagating freely to the right, and finally arriving at a transparent boundary condition on the right side.

In this numerical example, the computational domain for the Trefftz method is decomposed in the set of macro-elements $\mathcal{T}=\left\{T_{m}:=[m-1, m] \times[0,1]: m=1, \ldots, M\right\}$. The mesh used by the global Nédélec FEM is made of the union of Trefftz macro-elements meshes. In each of them, three triangular meshes $\mathcal{T}_{\nu}\left(T_{m}\right)$ based on spatial discretization steps $h=1 / N$ for $N=6,9,12$ are considered and an "optimal" Nédélec approximation order $p$ is chosen i.e $p=q+1$.

The aim of these simulations is a comparison of the relative $\mathrm{L}^{\infty}$-error

$$
e_{\infty}=100 \frac{\left\|\mathbf{E}-\mathbf{E}_{h}\right\|_{\infty}}{\|\mathbf{E}\|_{\infty}}
$$

induced by the Trefftz and the classical Nédélec FEM. It is known that this error is directly related to numerical dispersion and it is a relevant way to analyze the pollution effect. Here, the analytical solution of (52) is $\mathbf{E}(x)=e^{-i 2 \pi x} \mathbf{e}_{y}$. Empirically we observe that the relative maximum error as a function of duct length $L$ can be well approximated by a linear interpolation of the form

$$
e_{\text {interpolated }}=a L+b
$$

with $b>0$ and $a>0$.

The figure 8 (resp. 9 ) shows the results obtained with $q=1$ (resp. $q=2$ ) and a classical Nédélec FEM of order $p=1$ (resp. $p=2$ ). We can draw the following conclusions. The pollution error is always more important for the FEM. Comparing the curves, we see that both interpolation parameters $a$ and $b$ tend to be roughly one order of magnitude larger for the FEM than for the Trefftz method at comparable meshes and orders.
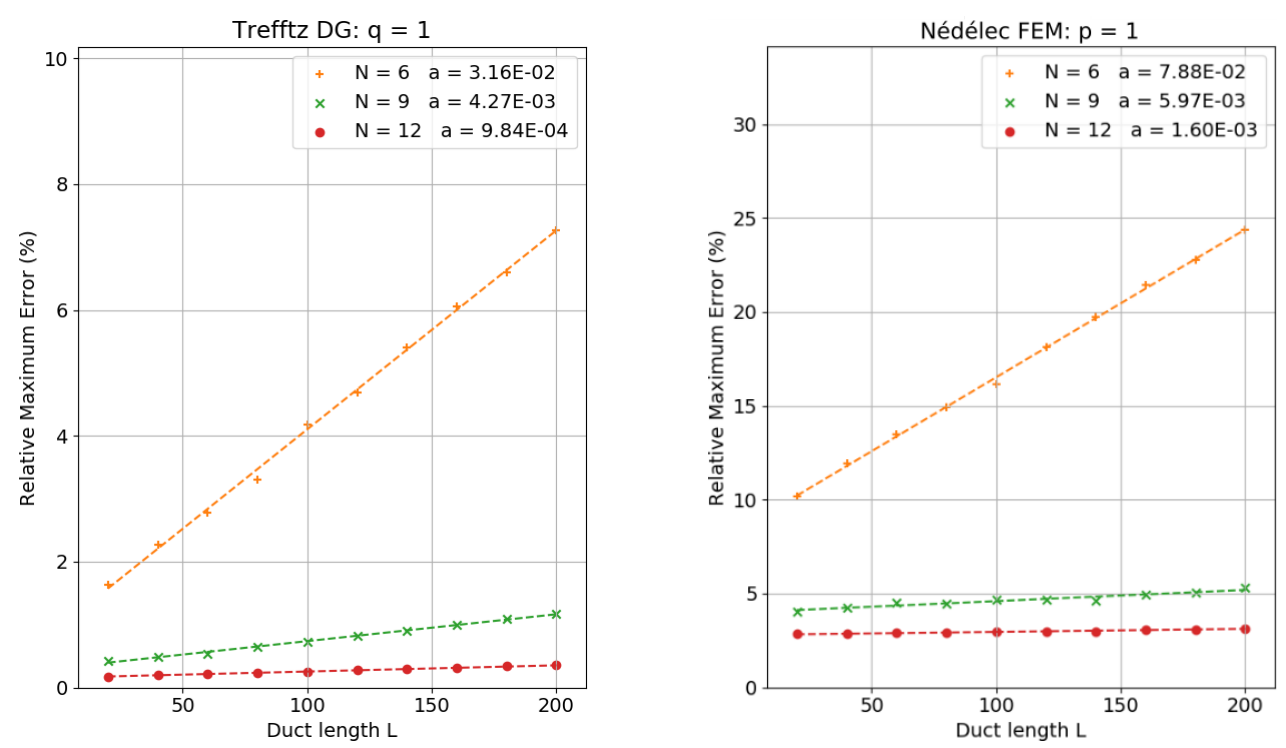

Fig. 8 Relative maximum order induced by the Trefftz DG method for $(q, p)=(1,2)$ and the classical Nédélec FEM of order $p=1$ in function of $L$. 

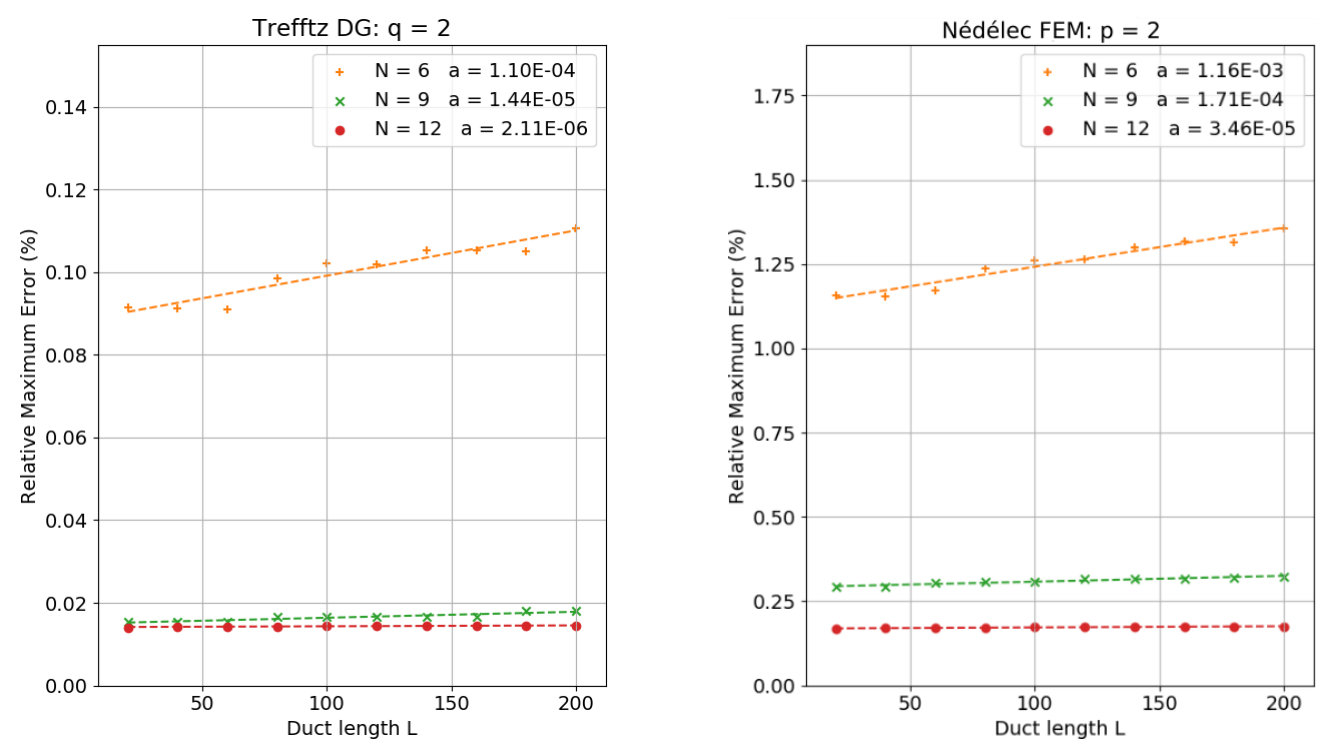

Fig. 9 Relative maximum order induced by the Trefftz DG method for $(q, p)=(2,3)$ and the classical Nédélec FEM of order $p=2$ in function of $L$.

\subsection{Illustrative examples}

We consider three examples to point out eur Trefftz method's accuracy and flexibility. Two of them consist of the computation of scattered field by bounded obstacles, see Fig 11 and Fig 12 . The other one is a heterogeneous duct problem. In all simulations, the domain is decomposed in a set $\mathcal{T}$ of macro-elements $T$. Triangular meshes $\mathcal{T}_{\nu}(T)$ are constructed by using spatial discretization steps $h_{\nu}$. From now on, we set the Trefftz order $q=2$ and the Nédélec FEM order $p=3$.

\subsubsection{Duct propagation with variable dielectric parameters}

In this example, we consider a duct problem with variable dielectric parameters defined by:

$$
\begin{array}{rlrl}
\nabla \times\left(\frac{1}{\mu_{r}} \nabla \times \mathbf{E}\right)-\varepsilon_{r} k_{0}^{2} \mathbf{E}=0 & & \text { in } \Omega=[0,6] \times[0,1], \\
\mathbf{n} \times(\mathbf{E} \times \mathbf{n}) & =0 & & \text { if } y=0 \text { or } y=1, \\
\mathbf{n} \times(\mathbf{E} \times \mathbf{n}) & =-1 & & \text { if } x=0, \\
i k_{0} Y \mathbf{n} \times(\mathbf{E} \times \mathbf{n})-\mathbf{n} \times\left(\nabla \times \frac{1}{\mu_{r}} \mathbf{E}\right) & =0 & & \text { if } x=6,
\end{array}
$$

where $k_{0}=3 \pi, Y=\sqrt{\varepsilon_{r} / \mu_{r}}$,

$$
\varepsilon_{r}(x, y)=\left\{\begin{array}{ll}
1 & \text { if } 0<x<2 \\
\frac{1}{2} & \text { if } 2<x<4 \\
2 & \text { if } 4<x<6
\end{array} \text { and } \mu_{r}(x, y)= \begin{cases}1 & \text { if } 0<x<2 \\
2 & \text { if } 2<x<4 \\
2 & \text { if } 4<x<6\end{cases}\right.
$$

For this simulation, the Trefftz approximation is characterized by the following table:

\begin{tabular}{|c|c|}
\hline$\Omega$ & {$[0,6] \times[0,1]$} \\
\hline $\mathcal{T}$ & $\left\{T_{m}:=[m, m+2] \times[0,1]: m=0,1,2\right\}$ \\
\hline$h_{\nu}$ & $1 / 12$ \\
\hline$(q, p)$ & $(2,3)$ \\
\hline
\end{tabular}

We recall the definition of the relative wave travelling speed and the relative impedance associated to a dielectric media:

$$
c_{r}=\frac{1}{\sqrt{\mu_{r} \varepsilon_{r}}} \text { and } Z_{r}=\sqrt{\frac{\mu_{r}}{\varepsilon_{r}}} .
$$


Now, we use the first macro-element (in blue in Fig. 10 where $c_{r}=Z_{r}=1$ as a reference. In the second macro-element (in red in Fig. 10, we have $c_{r}=1$ and $Z_{r}=2$ and physically, we must observe, in comparison to the first region, a doubling of the wave amplitude without modification of the wavenumber. In the third macro-element (in yellow in Fig. 10), we have $c_{r}=1 / 2$ and $Z_{r}=1$ and this situation leads to a doubling of the wavenumber without modification of the wave amplitude. Blue, red and yellow macro-elements in figure 10 are the meshes of the Nédélec local solver.

The figure 10 shows the imaginary part of the numerical solution computed by our Trefftz scheme. We observe that the desired physical behaviour imposed with amplitude and frequency changes by (55), is correctly reproduced.

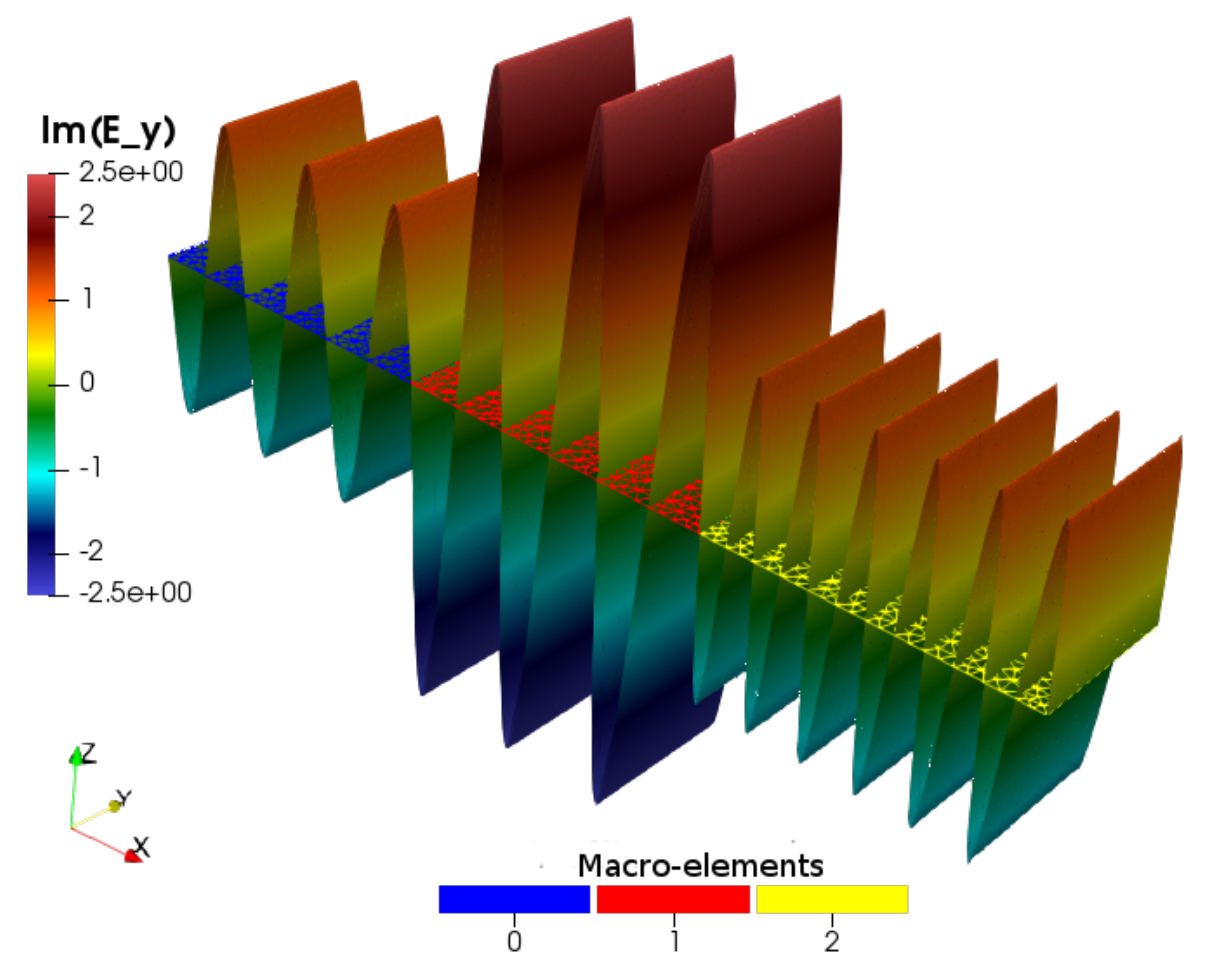

Fig. 10 A plane wave generated on the left edge (macro-element 0) propagating towards the right (towards macro-element 2). On macro-element 0: reference plane wave. On macro-element 1: twice the amplitude. On macro-element 2: twice the wavenumber.

\subsubsection{Scattering by a perfectly conducting disk and a L-shaped obstacle}

In this part, we present two test-cases which model the scattering of an incident plane wave by perfectly conducting obstacles in an unbounded domain. Meshes $\mathcal{T}_{\nu}(T)$ are constructed from two spatial discretization steps $h_{\nu}^{o b s}$, close to the obstacle, and $h_{\nu}^{e x t}$, close to the exterior boundary of the computational domain. We set the dielectric parameters to $\varepsilon_{r}=\mu_{r}=1$ and the wavenumber to $k_{0}=10 \pi$.

The first obstacle is smooth and convex and corresponds to a disk $D(0, R)$. The perfectly conducting character of this latter is taken into account by imposing the homogeneous Dirichlet boundary condition, $\mathbf{n} \times \mathbf{E}=0$ on the circle $C(0, R)$.

For this simulation, the Trefftz approximation is characterized by the following table: 


\begin{tabular}{|c|c|}
\hline$\Omega$ & $([-1,1] \times[-1,1]) \backslash D(0, R)$, with $R=0.4$ \\
\hline $\mathcal{T}$ & 4 macro-elements $T$ (bottom right of Fig. 11$)$ \\
\hline$h_{\nu}^{\text {obs }}$ & $1 / 30$ \\
\hline$h_{\nu}^{\text {ext }}$ & $1 / 15$ \\
\hline$(q, p)$ & $(2,3)$ \\
\hline
\end{tabular}

The scattered and total electric fields computed by the Trefftz method are represented in Fig. 11. We observe that the method accurately computes the electromagnetic wave propagation in such situation. In particular, the "shadow" region in the total field on the right side of the disk is well restored. Moreover, the non-convex macro-elements such as those used in this example are well supported.
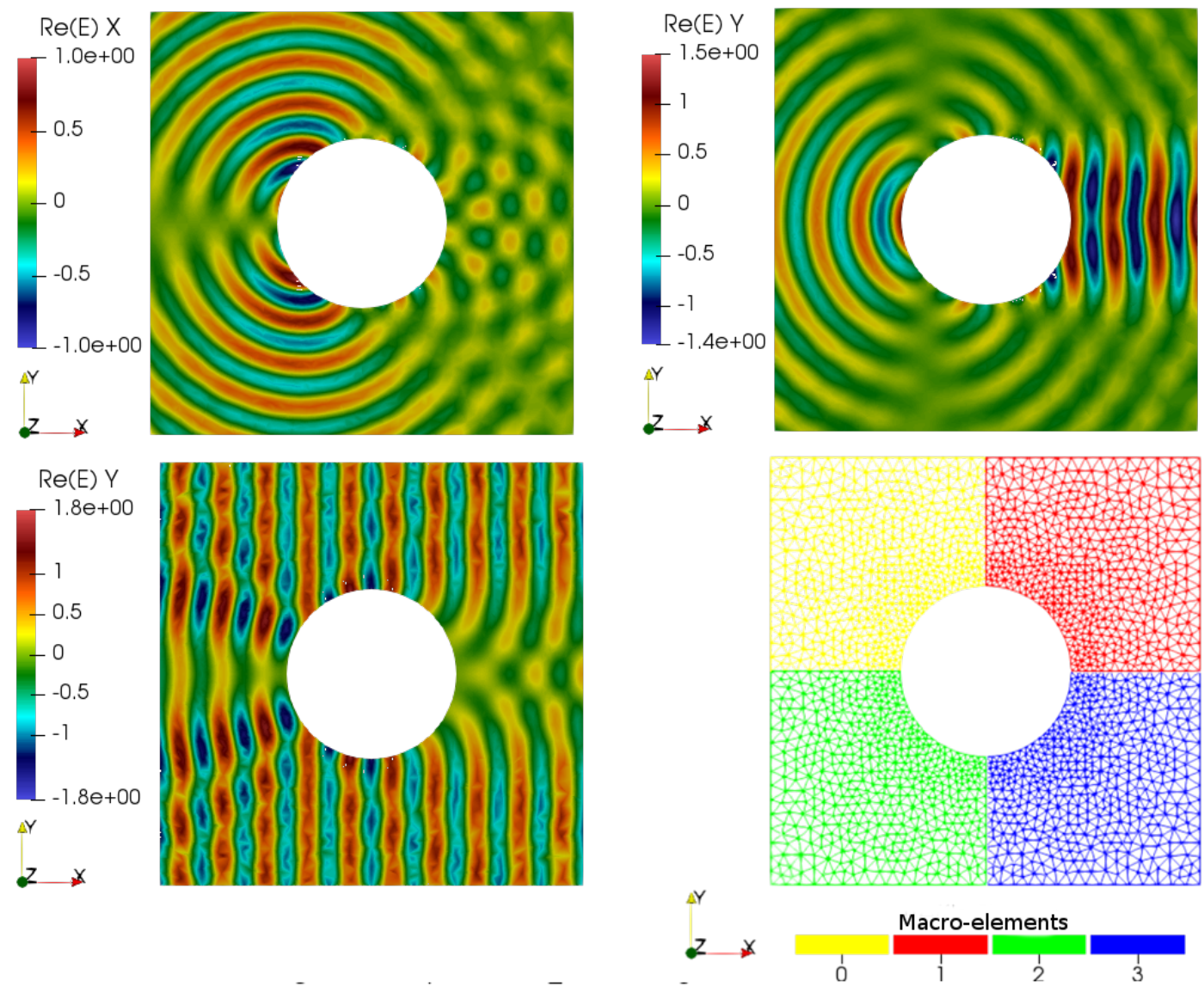

Fig. 11 On top left: scattered field (x-component). On top right: scattered field (y-component). On bottom left: total field (ycomponent). On bottom right: colour macro-elements with embedded FEM triangular mesh, refined close to the circle.

The second experiment uses the $L$-shaped obstacle $\Omega_{L}$ described in Fig. 12, which has a length and height of 1 , and a thickness of 0.2 . It leads to a non-convex and non-smooth problem whose approximation is defined by the following table:

\begin{tabular}{|c|c|}
\hline$\Omega$ & $([-1,1] \times[-1,1]) \backslash \Omega_{L}$ \\
\hline $\mathcal{T}$ & 12 macro-elements $T$ (top of Fig. 12$)$ \\
\hline$h_{\nu}^{\text {obs }}$ & $1 / 30$ \\
\hline$h_{\nu}^{\text {ext }}$ & $1 / 15$ \\
\hline$(q, p)$ & $(2,3)$ \\
\hline
\end{tabular}


The numerical solution represented in the figures on bottom of Fig. 12 shows that the proposed Trefftz method seems to work well in presence of singularities and to accurately compute the interference phenomenon which takes place in the trapping region induced by non-convexity of the obstacle according to classic electromagnetic theory. Moreover, this example gives a good idea of the method flexibility in terms of computational domain partitioning by using macro-elements.
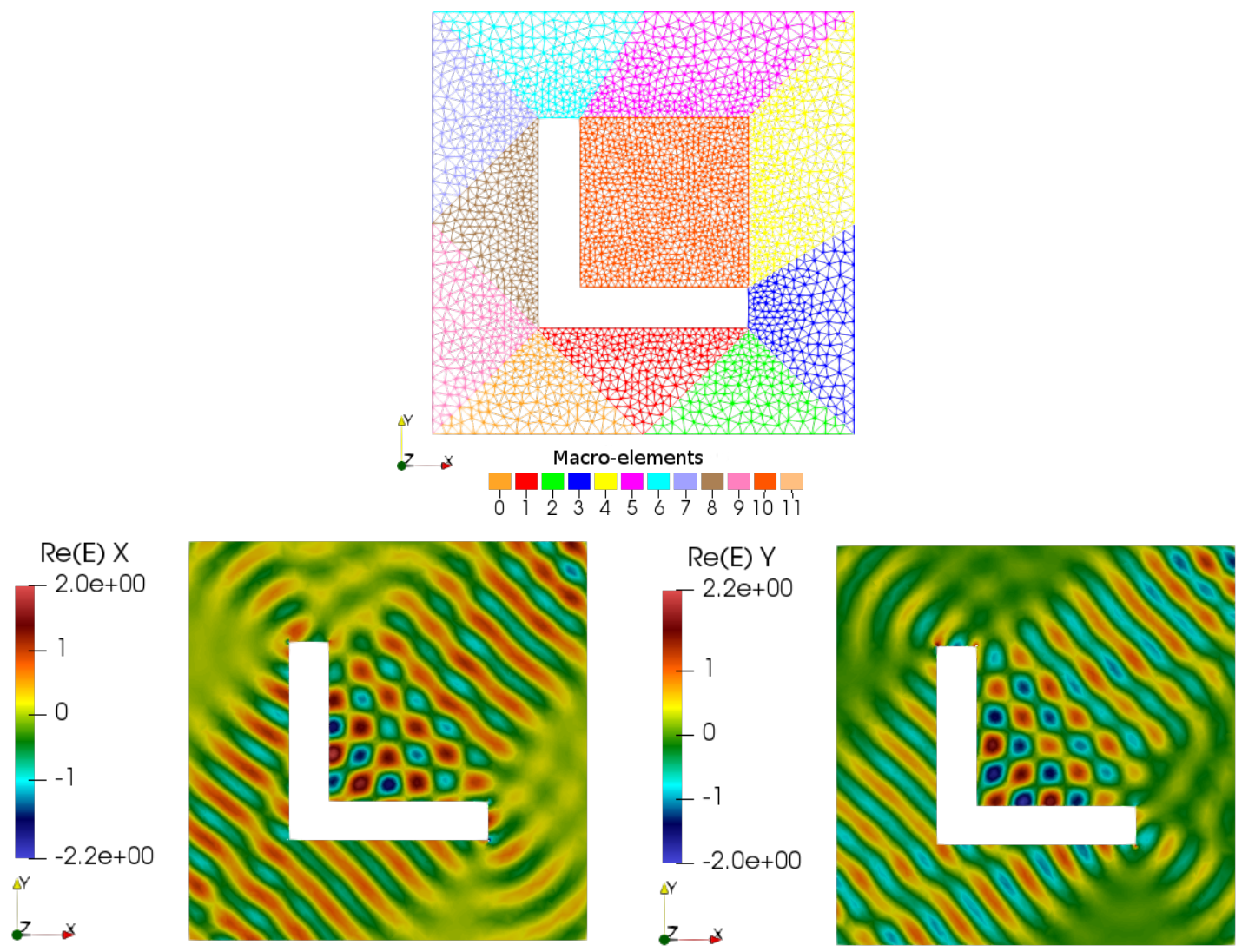

Fig. 12 On the top: $\mathcal{T}$ and $\mathcal{T}_{\nu}(T)$ associated to the L-shaped computational domain. On the bottom: $\mathrm{x}$ and $\mathrm{y}$ - components of the real part of the numerical solution.

\section{Conclusion}

In this paper, we have presented a Trefftz method associated to a Nédélec FEM approximation for a two dimensional Maxwell problem. As highlighted in our numerical analysis, a super-convergence phenomenon appears. This crucial aspect points out the "performance" of such a method. In a similar way, the propagation of electromagnetic waves through domains with obstacles emphasizes its robustness. This encourages us to consider a three dimensional implementation. However, these two dimensional cases are not really representative of three dimensional simulations which are more complex to implement.

Trefftz methods provide a particularly adapted framework for enriched Galerkin method in the context of multi-scale modeling as presented by Dauge et al. at the Enumath 2019 conference. This question is of particular importance for Maxwell equations. We are rather convinced that the proposed formulation is perfectly adapted to this type of problems.

The iterative solution of Trefftz problems is not dealt with in this paper. This seems to be a good alternative to efficient preconditioner like in [42. Testing GMRES solver [37] seems a good question for a future research program but is mostly relevant for three dimensional problems.

\section{References}

1. M. Ainsworth. Dispersive and dissipative behaviour of high order discontinuous Galerkin finite element methods. Journal of Computational Physics, 198:106-130, 2004.

2. M. Ainsworth. Dispersive properties of high-order Nédélec/edge element approximation of the timeharmonic maxwell equations. Philosophical Transactions of the Royal Society of London. Series A: Mathematical, Physical and Engineering Sciences, 362(1816):471-491, 2004. 
3. M. Ainsworth, P. Monk, and W. Muniz. Dispersive and dissipative properties of discontinuous Galerkin finite element methods for the second-order wave equation. Journal of Scientific Computing, 27(1-3):5-40, 2006.

4. I. Babuška and J. M. Melenk. The partition of unity method. International journal for numerical methods in engineering, 40(4):727-758, 1997.

5. H. Barucq, A. Bendali, M. Fares, V. Mattesi, and S. Tordeux. A symmetric DG formulation based on a local boundary element method for the solution of the Helmholtz equation. Journal of Computational Physics, 330:1069-1092, 2017.

6. O. P. Bruno and L. A. Kunyansky. A fast, high-order algorithm for the solution of surface scattering problems: basic implementation, tests, and applications. Journal of Computational Physics, 169(1):80-110, 2001.

7. A. Buffa and P. Monk. Error estimates for the ultra weak variational formulation of the Helmholtz equation. Mathematical Modelling and Numerical Analysis, 42(6):925-940, 2008.

8. F. Cakoni, D. Colton, and P. Monk. The electromagnetic inverse-scattering problem for partly coated Lipschitz domains. Proceedings of the Royal Society of Edinburgh Section A: Mathematics, 134(4):661$682,2004$.

9. O. Cessenat. Application d'une nouvelle formulation variationnelle aux équations d'ondes harmoniques. Problèmes d'Helmholtz 2D et de Maxwell 3D. PhD thesis, University of Paris XI Dauphine, 1996.

10. O. Cessenat and B. Després. Application of an ultra weak variational formulation of elliptic PDE to the two-dimensional Helmholtz problem. SIAM J. Num. Analysis, 35(1):255-299, 1998.

11. S. Congreve, J. Gedicke, and I. Perugia. Numerical investigation of the conditioning for plane wave discontinuous galerkin methods. In European Conference on Numerical Mathematics and Advanced Applications, pages 493-500. Springer, 2017.

12. E. Darve. The fast multipole method: numerical implementation. Journal of Computational Physics, 160(1):195-240, 2000.

13. B. Després. Sur une formulation variationnelle ultra-faible. Comptes Rendus de l'Académie des Sciences, Série I 318:939-944, 1994.

14. VJ Ervin. RTK and BDMK on triangles. Computers \& Mathematics with Applications, 64(8):2765-2774, 2012.

15. G. Gabard. Discontinuous Galerkin methods with plane waves for time-harmonic problems. Journal of Computational Physics, 225:1961-1984, 2007.

16. P. Gamallo and R. J. Astley. A comparison of two Trefftz-type methods: The ultraweak variational formulation and the least-squares method, for solving shortwave 2-D Helmholtz problems. International Journal for Numerical Methods in Engineering, 71:406-432, 2007.

17. C. Gittelson and R. Hiptmair. Dispersion analysis of plane wave discontinuous methods. International Journal for Numerical Methods in Engineering, 98(5):313-323, 2014.

18. C. J. Gittelson, R. Hiptmair, and I. Perugia. Plane wave discontinuous Galerkin methods: analysis of the $h$-version. Mathematical Modelling and Numerical Analysis, 43:297-331, 2009.

19. R. Hiptmair, A. Moiola, and I. Perugia. A survey of trefftz methods for the helmholtz equation. In Building bridges: connections and challenges in modern approaches to numerical partial differential equations, pages 237-279. Springer, 2016.

20. R. Hiptmair, A. Moiola, I. Perugia, and C. Schwab. Approximation by harmonic polynomials in starshaped domains and exponential convergence of Trefftz $h p$-dgfem. Mathematical Modelling and Numerical Analysis, 48:727-752, 2014.

21. C. Hofreither. A Non-standard Finite Element Method using Boundary Integral Operators. PhD thesis, J. Kepler University, Linz, 2012.

22. C. Hofreither, U. Langer, and S. Weißer. Convection-adapted BEM-based FEM. ZAMM-Journal of Applied Mathematics and Mechanics/Zeitschrift für Angewandte Mathematik und Mechanik, 96(12):1467-1481, 2016.

23. P. Monk I. Perugia, D. Schötzau. Stabilized interior penalty methods for the time-harmonic Maxwell equations. Comput. Methods Appl. Mech. Engrg., 191:4675-4697, 2002.

24. F. Ihlenburg and I. Babuska. Finite element solution of the Helmholtz equation with high wave number part i: the h-version of the FEM. Computers Math. Applic., 30(9):9-37, 1995.

25. F. Ihlenburg and I. Babuska. Finite element solution of the Helmholtz equation with high wave number part ii: the h-p version of the FEM. SIAM J. Numer. Anal., 34(1):315-358, 1997.

26. J. M. Jin. The Finite Element Method in Electromagnetics, Second Edition. John Wiley \& Sons, New York, 2002.

27. T. Luostari, T. Huttunen, and P. Monk. Improvements for the ultra weak variational formulation. International Journal for Numerical Methods in Engineering, 94(6):598-624, 2013. 
28. F. Magoules, F.-X. Roux, and S. Salmon. Optimal discrete transmission conditions for a non-overlapping domain decomposition method for the Helmholtz equation. SIAM Journal on Scientific Computing, 25(5):1497-1515, 2004.

29. W. McLean. Strongly Elliptic Systems and Boundary Integral Equations. Cambridge University Press, Cambridge university press, 2000.

30. J. M. Melenk, A. Parsania, and S. Sauter. General DG-Methods for highly indefinite Helmholtz problems. J. Sci. Comput., 57:536-581, 2013.

31. A. Moiola, R. Hiptmair, and I. Perugia. Plane wave approximation of homogeneous Helmholtz solutions. Z. Angew. Math. Phys., 62:809-837, 2011.

32. P. Monk. Finite Element Methods for Maxwell's Equations. Numerical Analysis and Scientific Computations. Clarendon Press, 2003.

33. P. Monk and D.-Q. Wang. A least-squares method for the helmholtz equation. Computer Methods in Applied Mechanics and Engineering, 175(1-2):121-136, 1999.

34. J.-C. Nédélec. Mixed finite elements in $\mathbb{R}^{3}$. Numer. Math., 35:315-341, 1980.

35. J.-C. Nédélec. A new family of mixed finite elements in $\mathbb{R}^{3}$. Numer. Math., 50:57-81, 1986.

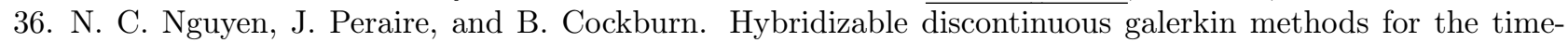
harmonic maxwell's equations. Journal of Computational Physics, 230(19):7151-7175, 2011.

37. Y. Saad. Iterative Methods for Sparse Linear Systems. PWS Pblishing Company, Boston, 1996.

38. S. A. Sauter and C. Schwab. Boundary Element Methods. Springer-Verlag, Berlin-Heidelberg, 2011.

39. T. B. A. Senior and J. L. Volakis. Approximate Boundary Conditions in Electromagnetics. IEE Electromagnetic Waves Series 41. IEE Press, New York, 1995.

40. K. Y. Sze and G. H. Liu. Hybrid-Trefftz six-node triangular finite element models for Helmholtz problems. Computational Mechanics, 46(6):455-470, 2010.

41. A. Taflove. Computational Electrodynamics. The Finite-Difference Time-Domain Method. Artech house, Inc., Norwood, MA 02062, 1995.

42. A. Vion and C. Geuzaine. Double sweep preconditioner for optimized Schwarz methods applied to the Helmholtz problem. J. Comput. Phys., 266:171-190, 2014.

43. D. Wang, R. Tezaur, J. Toivanen, and C. Ferhat. Overview of the discontinuous enrichment method, the ultra-weak variational formulation, and the partition of unity method for the acoustic scattering in the medium frequency regime and performance comparisons. International Journal for Numerical Methods in Engineering, 89:403-417, 2012.

44. N. Zerbib. Méthodes de Sous-Structuration et de Décomposition de Domaine pour la Résolution des Équations de Maxwell : Application au Rayonnement d'antenne dans un Environnement Complexe. PhD thesis, National Institute for Applied Sciences (INSA), INSA Toulouse, 2006.

45. K. Zhao, M. N. Vouvakis, and J.-F. Lee. The adaptive cross approximation algorithm for accelerated method of moments computations of emc problems. IEEE transactions on electromagnetic compatibility, 47(4):763-773, 2005.

46. L. Zhu, E. Burman, and H. Wu. Continuous interior penalty finite element method for helmholtz equation with high wave number: One dimensional analysis. preprint available at arXiv:1211.1424. 